\title{
Robust Fault Estimation for a Class of T-S Fuzzy Singular Systems with Time-Varying Delay via Improved Delay Partitioning Approach
}

\author{
Chao Sun, ${ }^{1,2}$ FuLi Wang, ${ }^{1,3}$ and XiQin $\mathrm{He}^{2}$ \\ ${ }^{1}$ College of Information Science and Engineering, Northeastern University, Shenyang, Liaoning 110819, China \\ ${ }^{2}$ College of Sciences, University of Science and Technology Liaoning, Anshan, Liaoning 114051, China \\ ${ }^{3}$ State Key Laboratory of Synthetical Automation of Process Industries, Northeastern University, \\ Shenyang, Liaoning 110819, China \\ Correspondence should be addressed to Chao Sun; chao_sun@163.com
}

Received 12 August 2015; Revised 4 January 2016; Accepted 6 January 2016

Academic Editor: Kalyana C. Veluvolu

Copyright (C) 2016 Chao Sun et al. This is an open access article distributed under the Creative Commons Attribution License, which permits unrestricted use, distribution, and reproduction in any medium, provided the original work is properly cited.

\begin{abstract}
The problem of delay-dependent robust fault estimation for a class of Takagi-Sugeno (T-S) fuzzy singular systems is investigated. By decomposing the delay interval into two unequal subintervals and with a new and tighter integral inequality transformation, an improved delay-dependent stability criterion is given in terms of linear matrix inequalities (LMIs) to guarantee that the fuzzy singular system with time-varying delay is regular, impulse-free, and stable firstly. Then, based on this criterion, by considering the system fault as an auxiliary disturbance vector and constructing an appropriate fuzzy augmented system, a fault estimation observer is designed to ensure that the error dynamic system is regular, impulse-free, and robustly stable with a prescribed $H_{\infty}$ performance satisfied for all actuator and sensor faults simultaneously, and the obtained fault estimates can practically better depict the size and shape of the faults. Finally, numerical examples are given to show the effectiveness of the proposed approach.
\end{abstract}

\section{Introduction}

The demand for increased productivity leads to more challenging operating conditions for many modern engineering systems. The issue of fault detection and isolation (FDI) algorithms in dynamic systems and their applications to a wide range of industrial processes have been an active research area over the past two decades, as can be seen, in survey papers [1] for linear systems, [2] for multimodels representation and $[3,4]$ for nonlinear systems. By using FDI procedures, the reliability can be achieved by faulttolerant control, which relies on early detection and isolation of faults. So FDI have become a popular topic and received considerable attention. However, it is generally difficult, in practice engineering, to obtain the exact information of the size of system fault from an FDI strategy only because of the existence of model uncertainties, time delays, and disturbances [5]. As pointed out in [6], accurate and timely fault estimation is an important antecedent for satisfactory control reconfiguration. Therefore, the problem of fault estimation has stirred renewed research interest, and a variety of fault estimation approaches have been developed in the literatures; see, for example, [7-11] and the references therein.

On the other hand, singular systems have been extensively studied in the past years due to the fact that singular systems better describe physical systems than state-space ones, especially the T-S fuzzy singular systems, because they can combine the flexibility of fuzzy logic theory and fruitful linear singular system theory into a unified framework to approximate complex nonlinear singular systems. In fact, singular systems can be found in electrical circuits, economic systems, moving robots, and many other systems. Recently, many results about fault estimation have been reported on analysis and design of singular systems. For instance, by using online learning methodology, [12] proposed a fault estimation method for continuous-time nonlinear singular systems. Reference [7] used generalized unknown input observer to deal with the robust fault detection problem for 
linear singular systems. Reference [13] deals with actuator fault estimation for a class of discrete-time linear parametervarying singular systems. Reference [14] designed a robust fault detection filter for a class of nonlinear singular systems described by linear parameter-varying form with global Lipschitz term. However, it is known that time delays are frequently encountered in various engineering and communication systems, and a time delay in a dynamical system is often a primary source of instability and performance degradation. Therefore, it is important to develop fault estimation methods for time delay singular systems. But the fault diagnosis for singular systems with time-varying delay has not been well investigated yet [7, 12-14]. More recently, [15] proposed a fault detection, isolation, and estimation scheme via unknown input proportional integral observers for linear descriptor systems. Reference [8] investigated fault detection for discrete-time switched singular systems with time-varying delays. It should be noticed that [8] deals with discrete-time switched singular systems while this paper focuses on the fuzzy continuous-time case. In [16], discretetime T-S systems with sensor faults are first formulated as a descriptor representation, and then a fault detection filter is designed based on the obtained descriptor system. However, this paper studies fault detection for regular systems by using the technique of descriptor systems. In [17], the author proposed a k-step fault estimation method for T-S fuzzy time delay system that only deals with regular systems. Moreover, our paper considers how to estimate the actuator and sensor faults simultaneously while attenuating the influence of the disturbance noise, which is not considered in [17]. It is known that singular system representation is a generalization of the regular system. Therefore, the proposed method is more general than that in $[16,17]$.

The aim of this paper is to develop a robust fault estimation method for a class of T-S fuzzy singular systems with time delays. The basic idea is to construct an augmented system by taking the fault as auxiliary disturbance vector and then design a fault estimation observer based on this augmented system. The main contribution of the proposed method lies in the following aspects. First, without ignoring any useful terms in the Lyapunov-Krasovskii functional (LKF), by decomposing the delay interval into two unequal subintervals, a new LKF is constructed; then the free weighting matrices approach is introduced to develop a new delay-dependent stability criteria, which ensure that the considered system is regular, impulse-free, and stable. Compared with some existing results, the approach to be proposed in this paper can be expected to give better results. Second, a new robust fault estimation observer with a novel structure is proposed for $\mathrm{T}$ $S$ fuzzy singular systems with time delays and actuator and sensor faults simultaneously, which is the main contribution of this paper. The proposed observer can be designed by solving a set of linear matrix inequalities and to attenuate the effect of unknown disturbance, fault variation on fault estimation. The effectiveness of the method is illustrated by some numerical examples.

The rest of this paper is organized as follows. The system description and preliminaries are presented in Section 2. Section 3 presents the main results on new stability criteria of fuzzy singular systems with time-varying delays and robust fault estimation observer design scheme. In Section 4, simulation results of numerical examples are presented to demonstrate the effectiveness and merits of the proposed methods. Finally, Section 5 concludes the paper.

Notations. Throughout the paper, $\mathbb{R}^{n}$ denotes the $n$ dimensional real Euclidean space; $I$ denotes the identity matrix; the superscripts $T$ and -1 stand for the matrix transpose and inverse, respectively; notation $X>0(X \geq 0)$ means that matrix $X$ is real symmetric positive definite (positive semidefinite); $\|\cdot\|$ is the spectral norm. If not explicitly stated, all matrices are assumed to have compatible dimensions for algebraic operations. The symbol “*” stands for matrix block induced by symmetry; $\operatorname{sym}(X)$ stands for $X+X^{T}$.

\section{System Description and Preliminaries}

Consider a nonlinear singular system which can be represented by the following extended T-S fuzzy time delay model with external disturbance, actuator and sensor faults, simultaneously.

Plant Rule $i$. If $\xi_{1}(t)$ is $M_{i 1}$ and $\ldots$ and $\xi_{p}(t)$ is $M_{i p}$, then

$$
\begin{aligned}
E \dot{x}(t)= & A_{i} x(t)+A_{\tau i} x(t-\tau(t))+B_{i} u(t)+B_{f i} f(t) \\
& +B_{d i} d(t), \\
y(t)= & C_{i} x(t)+C_{\tau i} x(t-\tau(t))+D_{i} u(t)+D_{f i} f(t) \\
& +D_{d i} d(t), \\
x(t)= & \phi_{i}(t), \quad \forall t \in\left[-\tau_{2}, 0\right], i=1,2, \ldots, r,
\end{aligned}
$$

where $x(t) \in \mathbb{R}^{n}$ is the state vector, $u(t) \in \mathbb{R}^{q}$ denotes the input vector, and $y(t) \in \mathbb{R}^{l}$ stands for the system output vector. $d(t) \in \mathbb{R}^{m}$ is the exogenous disturbance input that belongs to $L_{2}[0, \infty) ; f(t) \in \mathbb{R}^{q}$ represents the possible fault. The matrix $E \in \mathbb{R}^{n \times n}$ is a constant matrix, which may be singular; that is, $\operatorname{rank}(E)=g \leq n . A_{i}, A_{\tau i}, B_{i}, B_{f i}, B_{d i}, C_{i}$, $C_{\tau i}, D_{i}, D_{f i}$, and $D_{d i}$ are constant real matrices of appropriate dimensions. It is assumed that the pairs $\left(E, A_{i}, C_{i}\right)$ are of full column rank, where $i=1,2, \ldots, r . \xi_{1}(t), \ldots, \xi_{p}(t)$ are the premise variables, $M_{i j}(i=1,2, \ldots, r, j=1,2, \ldots, p)$ are fuzzy sets, and $\phi_{i}(t)$ is a vector-valued initial continuous function defined on the interval $\left[-\tau_{2}, 0\right]$. In this paper, it is also assumed that the premise variables do not depend on the input variables $u(t) ; \tau(t)$ is the time-varying delay and satisfies

$$
\begin{aligned}
& \tau_{1} \leq \tau(t) \leq \tau_{2}, \\
& 0 \leq \dot{\tau}(t) \leq \tau_{D} .
\end{aligned}
$$

Then, by fuzzy blending, the overall fuzzy singular system model is given by

$$
\begin{aligned}
& E \dot{x}(t)=\sum_{i=1}^{r} \mu_{i}(\xi(t))\left\{A_{i} x(t)+A_{\tau i} x(t-\tau(t))\right. \\
& \left.+B_{i} u(t)+B_{f i} f(t)+B_{d i} d(t)\right\}
\end{aligned}
$$




$$
\begin{aligned}
y(t) & =\sum_{i=1}^{r} \mu_{i}(\xi(t))\left\{C_{i} x(t)+C_{\tau i} x(t-\tau(t))+D_{i} u(t)\right. \\
+ & \left.D_{f i} f(t)+D_{d i} d(t)\right\}, \\
x(t) & =\sum_{i=1}^{r} \mu_{i}(\xi(t)) \phi_{i}(t), \quad \forall t \in\left[-\tau_{2}, 0\right]
\end{aligned}
$$

where the fuzzy basis functions are given by

$$
\mu_{i}(\xi(t))=\frac{\beta_{i}(\xi(t))}{\sum_{j=1}^{r} \beta_{j}(\xi(t))}, \quad \beta_{i}(\xi(t))=\prod_{i=1}^{p} M_{i j}(\xi(t))
$$

where $M_{i j}\left(\xi_{j}(t)\right)$ represents the grade of membership of $\xi_{j}(t)$ in $M_{i j}$. Here, it is easy to find that for all $t>0$, we have

$$
\begin{aligned}
& \beta_{i}(\xi(t)) \geq 0, \quad(i=1,2, \ldots, r), \sum_{j=1}^{r} \beta_{j}(\xi(t))>0 \\
& \mu_{i}(\xi(t)) \geq 0, \quad(i=1,2, \ldots, r), \sum_{j=1}^{r} \mu_{j}(\xi(t))=1
\end{aligned}
$$

For convenience of notations, in the sequel, we denote

$$
\begin{aligned}
A(t) & =\sum_{i=1}^{r} \mu_{i}(\xi(t)) A_{i} \\
A_{\tau}(t) & =\sum_{i=1}^{r} \mu_{i}(\xi(t)) A_{\tau i} \\
B(t) & =\sum_{i=1}^{r} \mu_{i}(\xi(t)) B_{i} \\
B_{f}(t) & =\sum_{i=1}^{r} \mu_{i}(\xi(t)) B_{f i} \\
B_{d}(t) & =\sum_{i=1}^{r} \mu_{i}(\xi(t)) B_{d i} \\
C(t) & =\sum_{i=1}^{r} \mu_{i}(\xi(t)) C_{i} \\
D_{d}(t) & =\sum_{i=1}^{r} \mu_{i}(\xi(t)) D_{d i} \\
D_{f}(t) & =\sum_{i=1}^{r} \mu_{i}(\xi(t)) C_{\tau i} \\
D(t) & =\sum_{i=1}^{r} \mu_{i}(\xi(t)) D_{i} \\
D_{i} & (\xi(t)) D_{f i} \\
D_{i} & \\
D_{i} &
\end{aligned}
$$

Then, we can rewrite system (3) as

$$
\begin{aligned}
E \dot{x}(t)= & A(t) x(t)+A_{\tau}(t) x(t-\tau(t))+B(t) u(t) \\
& +B_{f}(t) f(t)+B_{d}(t) d(t), \\
y(t)= & C(t) x(t)+C_{\tau}(t) x(t-\tau(t))+D(t) u(t) \\
& +D_{f}(t) f(t)+D_{d}(t) d(t), \\
x(t)= & \phi(t), \quad t \in\left[-\tau_{2}, 0\right] .
\end{aligned}
$$

Before proceeding further, we will introduce some definitions and assumptions to be needed in the development of main results throughout this paper. Consider an unforced singular time delay system described by

$$
\begin{aligned}
E \dot{x}(t) & =A x(t)+A_{\tau} x(t-\tau(t)) \\
x(t) & =\phi(t), \quad t \in[-\tau, 0] .
\end{aligned}
$$

Definition 1 (see [18]). (1) The pair $(E, A)$ is said to be regular if $\operatorname{det}(z E-A)$ is not identically zero.

(2) The pair $(E, A)$ is said to be impulse-free if $\operatorname{deg}(\operatorname{det}(z E-A))=\operatorname{rank}(E)$.

(3) The pair $(E, A)$ is said to be stable, if all roots of $\operatorname{det}(z E-A)=0$ lie inside the unit disk with center at the origin.

(4) The delayed singular system (8) is said to be admissible if the pair $(E, A)$ is regular, impulse-free, and stable.

Definition 2 (see [19]). (1) The singular system (8) is said to be regular and impulse-free if the pair $(E, A)$ is regular and impulse-free.

(2) The singular system (8) is said to be asymptotically stable, if for any $\varepsilon>0$, there exists a scalar $\delta(\varepsilon)>0$ such that, for any compatible initial conditions, $\phi(t)$ with $\sup _{-\tau(t) \leq t \leq 0}\|\phi(t)\|<\delta(\varepsilon)$; the solution $x(t)$ of (8) satisfies $\|x(t)\|<\varepsilon$ for $t \geq 0$ and $\lim _{t \rightarrow \infty} x(t)=0$.

The singular time delay system (8) may have an impulsive solution. However, the regularity and nonimpulse of $(E, A)$ guarantee the existence and uniqueness of impulse-free solution to $(8)$ on $[0, \infty)$.

Lemma 3 (see [20]). If a functional $V: C_{n}[-\tau, 0] \rightarrow \mathbb{R}$ is continuous and $x(t, \phi)$ is a solution to (8), one defines $\dot{V}(\phi)=$ $\lim _{h \rightarrow 0^{+}} \sup (1 / h)(V(x(t+h, \phi)-V(\phi)))$. Denote the system parameters of (8) as

$$
\left(E, A, A_{\tau}\right)=\left(\left[\begin{array}{ll}
I_{g} & 0 \\
0 & 0
\end{array}\right],\left[\begin{array}{ll}
A_{11} & A_{12} \\
A_{21} & A_{22}
\end{array}\right],\left[\begin{array}{ll}
A_{\tau 11} & A_{\tau 12} \\
A_{\tau 21} & A_{\tau 22}
\end{array}\right]\right) .
$$

Assume that the singular system (8) is regular and impulsefree, $A_{22}$ is invertible, and $\rho\left(A_{22}^{-1} A_{\tau 22}\right)<1$. Then, system (8) is stable if there exists positive numbers $\alpha, \mu, \nu$, and a continuous function; $V: C_{n}[-\tau, 0] \rightarrow \mathbb{R}$, such that

$$
\begin{gathered}
\mu\left\|\phi_{1}(0)\right\|^{2} \leq V(\phi) \leq \nu\|\phi\|^{2}, \\
\dot{V}\left(x_{t}\right) \leq-\alpha\left\|x_{t}\right\|^{2},
\end{gathered}
$$

where $x_{t}=x(t+\theta)$ with $\theta \in[-\tau, 0]$ and $\phi=\left[\begin{array}{ll}\phi_{1}^{T} & \phi_{2}^{T}\end{array}\right]$ with $\phi_{1} \in \mathbb{R}^{q}$. 
In order to address the main results, the following assumptions are made.

Assumption 4. Matrices $E$ and $C_{i}$ satisfy the following rank condition:

$$
\operatorname{rank}\left[\begin{array}{c}
E \\
C_{i}
\end{array}\right]=n
$$

Assumption 5. The triple matrix $\left(E, A_{i}, C_{i}\right)$ is $R$-detectable [21] for $\forall i=1,2, \ldots, r$; that is,

$$
\operatorname{rank}\left[\begin{array}{c}
s E-A_{i} \\
C_{i}
\end{array}\right]=n, \quad s \in C_{+} .
$$

Remark 6. Both Assumptions 4 and 5 are necessary conditions for the existence of the designed observer in the latter section. Similar assumptions can be also found in [21] and the references therein. Meanwhile, for T-S fuzzy system description (3), we can see that a general time-varying delay fuzzy singular system is considered in this paper, including possible actuator, sensor faults, and exogenous disturbance input simultaneously.

\section{Main Results}

3.1. Delay-Dependent Stability. In this subsection, we suggest developing a delay-dependent stability condition for the nominal unforced fuzzy singular system of (7), which can be written as

$$
\begin{aligned}
E \dot{x}(t) & =A(t) x(t)+A_{\tau}(t) x(t-\tau(t))+B_{d}(t) d(t), \\
y(t) & =C(t) x(t)+C_{\tau}(t) x(t-\tau(t))+D_{d}(t) d(t), \\
x(t) & =\phi(t), \quad t \in\left[-\tau_{2}, 0\right] .
\end{aligned}
$$

Theorem 7. For the given $\tau_{1}, \tau_{2}, \tau_{D}$, the free fuzzy singular system (13) with $d(t)=0$ is admissible for any timevarying delay $\tau(t)$ satisfying (2), if there exists a nonsingular matrix $P$, symmetric positive-definite matrices $Q_{1}>0$, $Q_{2}>0, S_{1}>0, S_{2}>0$, and $R>0$, and any appropriately dimensioned matrices $V=\left[\begin{array}{lllll}V_{1} & V_{2} & V_{3} & V_{4} & V_{5}\end{array}\right]$ and $W=\left[\begin{array}{lllll}W_{1} & W_{2} & W_{3} & W_{4} & W_{5}\end{array}\right]$, such that the following set of inequalities hold:

$$
\begin{aligned}
& E^{T} P=P^{T} E \geq 0, \\
& {\left[\begin{array}{cc}
\Psi_{i} & \left(\tau_{2}-\tau_{1}\right) V^{T} \\
* & -\left(\tau_{2}-\tau_{1}\right) R
\end{array}\right]<0,} \\
& {\left[\begin{array}{cc}
\Psi_{i} & \left(\tau_{2}-\tau_{1}\right) W^{T} \\
* & -\left(\tau_{2}-\tau_{1}\right) R
\end{array}\right]<0,}
\end{aligned}
$$

where

$$
\begin{aligned}
& \Psi_{i}=\Psi_{1 i}+\left(\tau_{2}-\tau_{1}\right) \Gamma_{1}^{T} R \Gamma_{1}, \\
& \Psi_{1 i} \\
& =\left[\begin{array}{ccccc}
\Psi_{11 i} & -V_{1}^{T} E & V_{1}^{T} E-W_{1}^{T} E & W_{1}^{T} E & P^{T} A_{\tau i} \\
* & \Psi_{22} & \Psi_{23} & W_{2}^{T} E-E^{T} V_{4} & -E^{T} V_{5} \\
* & * & \Psi_{33} & \Psi_{34} & E^{T} V_{5}-E^{T} W_{5} \\
* & * & * & \Psi_{44} & E^{T} W_{5} \\
* & * & * & * & -\varepsilon\left(1-\tau_{D}\right) Q_{1}
\end{array}\right]
\end{aligned}
$$

with

$$
\begin{aligned}
& \Psi_{11 i}=P^{T} A_{i}+A_{i}^{T} P+\varepsilon Q_{1}+Q_{2}, \\
& \Psi_{22}=S_{1}-Q_{2}-V_{2}^{T} E-E^{T} V_{2}, \\
& \Psi_{23}=V_{2}^{T} E-W_{2}^{T} E-E^{T} V_{3}, \\
& \Psi_{33}=S_{2}-S_{1}+\operatorname{sym}\left(V_{3}^{T} E-W_{3}^{T} E\right), \\
& \Psi_{34}=W_{3}^{T} E-E^{T} V_{4}-E^{T} W_{4}, \\
& \Psi_{44}=-S_{2}+W_{4}^{T} E+E^{T} W_{4} .
\end{aligned}
$$

Proof. The proof of this theorem is divided into two parts. The first one is concerned with the regularity and the impulsefree characterizations, and the second one treats the stability property of system (13).

Since $\operatorname{rank}(E)=g \leq n$, there must exist two invertible matrices $G \in \mathbb{R}^{n \times n}$ and $H \in \mathbb{R}^{n \times n}$ such that

$$
\widetilde{E}=G E H=\left[\begin{array}{ll}
I_{g} & 0 \\
0 & 0
\end{array}\right]
$$

Similar to (19), we define

$$
\begin{gathered}
\widetilde{A}_{i}=G A_{i} H=G\left[\begin{array}{ll}
A_{i 11} & A_{i 12} \\
A_{i 21} & A_{i 22}
\end{array}\right] H=\left[\begin{array}{cc}
\widetilde{A}_{i 11} & \widetilde{A}_{i 12} \\
\widetilde{A}_{i 21} & \widetilde{A}_{i 22}
\end{array}\right], \\
i=1,2, \ldots, r, \\
\widetilde{P}=G^{-T} P H=G^{-T}\left[\begin{array}{ll}
P_{11} & P_{12} \\
P_{21} & P_{22}
\end{array}\right] H=\left[\begin{array}{ll}
\widetilde{P}_{11} & \widetilde{P}_{12} \\
\widetilde{P}_{21} & \widetilde{P}_{22}
\end{array}\right] .
\end{gathered}
$$

Since $\Psi_{i}<0$ and $Q_{1}>0$ and $Q_{2}>0$ and $R>0$, we can formulate the following inequality easily:

$$
\digamma=A_{i}^{T} P+P^{T} A_{i}<0
$$

Then, pre- and postmultiplying $F<0$ by $H^{T}$ and $H$, respectively, (21) yields

$$
\widetilde{F}=\widetilde{A}_{i}^{T} \widetilde{P}+\widetilde{P}^{T} \widetilde{A}_{i}=\left[\begin{array}{cc}
\widetilde{F}_{11} & \widetilde{F}_{12} \\
* & \widetilde{A}_{i 22}^{T} \widetilde{P}_{22}+\widetilde{P}_{22}^{T} \widetilde{A}_{i 22}
\end{array}\right]<0 .
$$


Since $\widetilde{F}_{11}$ and $\widetilde{F}_{12}$ are irrelevant to the results of the following discussion, the real expression of these two variables is omitted here. From (22), it is easy to see that

$$
\widetilde{A}_{i 22}^{T} \widetilde{P}_{22}+\widetilde{P}_{22}^{T} \widetilde{A}_{i 22}<0
$$

Since $\mu_{i}(\xi(t)) \geq 0$ and $\sum_{i=1}^{r} \mu_{i}(\xi(t))=1$, we have

$$
\sum_{i=1}^{r} \mu_{i}(\xi(t))\left(\widetilde{A}_{i 22}^{T} \widetilde{P}_{22}+\widetilde{P}_{22}^{T} \widetilde{A}_{i 22}\right)<0 .
$$

This implies that $\sum_{i=1}^{r} \mu_{i}(\xi(t)) \widetilde{A}_{i 22}$ is nonsingular. Therefore, the unforced fuzzy singular system (13) is regular and impulse-free.

Next, we will show the stability of system (13). If conditions (15)-(16) hold, we have

$$
\begin{aligned}
& \sum_{i=1}^{r} \mu_{i}\left[\begin{array}{cc}
P^{T} A_{i}+A_{i}^{T} P+\varepsilon Q_{1}+Q_{2} & P^{T} A_{\tau i} \\
* & -\varepsilon\left(1-\tau_{D}\right) Q_{1}
\end{array}\right] \\
& \quad<0 .
\end{aligned}
$$

Premultiplying and postmultiplying the preceding inequality by

$$
\left[\begin{array}{llll}
0 & I & 0 & 0 \\
0 & 0 & 0 & I
\end{array}\right]
$$

and its transposes, respectively, we obtain

$$
\left[\begin{array}{cc}
\operatorname{sym}\left(P_{22}^{T}\left(\sum_{i=1}^{r} \mu_{i} A_{i 22}\right)\right)+\varepsilon Q_{122}+Q_{222} & P_{22}^{T}\left(\sum_{i=1}^{r} \mu_{i} A_{\tau i 22}\right) \\
* & -\varepsilon\left(1-\tau_{D}\right) Q_{122}
\end{array}\right]
$$

$<0$.

which implies that

$$
\begin{aligned}
& {\left[\begin{array}{cc}
\operatorname{sym}\left(P_{22}^{T}\left(\sum_{i=1}^{r} \mu_{i} A_{i 22}\right)\right)+\varepsilon Q_{122} & P_{22}^{T}\left(\sum_{i=1}^{r} \mu_{i} A_{\tau i 22}\right) \\
* & -\varepsilon\left(1-\tau_{D}\right) Q_{122}
\end{array}\right]} \\
& \quad<0 .
\end{aligned}
$$

It follows from $(1,1)$-block of $(28)$ that $A_{i 22}$ is invertible. Then, premultiplying and postmultiplying the preceding inequality by

$$
\left[-\left(\sum_{i=1}^{r} \mu_{i} A_{\tau i 22}\right)^{T}\left(\sum_{i=1}^{r} \mu_{i} A_{i 22}\right)^{-T} I\right]<0
$$

and its transpose, respectively, yield

$$
\begin{aligned}
& \left(\left(\sum_{i=1}^{r} \mu_{i} A_{i 22}\right)^{-1}\left(\sum_{i=1}^{r} \mu_{i} A_{\tau i 22}\right)\right)^{T} \\
& \cdot Q_{122}\left(\left(\sum_{i=1}^{r} \mu_{i} A_{i 22}^{-1}\right)\left(\sum_{i=1}^{r} \mu_{i} A_{\tau i 22}\right)\right)-\left(1-\tau_{D}\right) \\
& \cdot Q_{122}<0
\end{aligned}
$$

which shows that $\rho\left(\left(\sum_{i=1}^{r} \mu_{i} A_{i 22}\right)^{-1}\left(\sum_{i=1}^{r} \mu_{i} A_{\tau i 22}\right)\right)<1$ holds for all allowable $\mu_{i}$.

Now, let us choose the following Lyapunov-Krasovskii function as

$$
V(t)=V_{1}(t)+V_{2}(t)+V_{3}(t),
$$

where

$$
\begin{aligned}
V_{1}(t)= & x^{T}(t) E^{T} P x(t) \\
V_{2}(t)= & \varepsilon \int_{t-\tau(t)}^{t} x^{T}(s) Q_{1} x(s) d s \\
& +\int_{t-\tau_{1}}^{t} x^{T}(s) Q_{2} x(s) d s \\
& +\int_{t-\tau_{\rho}}^{t-\tau_{1}} x^{T}(s) S_{1} x(s) d s \\
& +\int_{t-\tau_{2}}^{t-\tau_{\rho}} x^{T}(s) S_{2} x(s) d s, \\
V_{3}(t)= & \int_{-\tau_{2}}^{-\tau_{1}} \int_{t+\theta}^{t} \dot{x}^{T}(s) E^{T} R E \dot{x}(s) d s d \theta
\end{aligned}
$$

where the unknown matrices $P, Q_{1}>0, Q_{2}>0, S_{1}>0$, $S_{2}>0$, and $R>0$ are to be determined, and $\tau_{\rho}=\tau_{1}+\rho \delta$, $\delta=\tau_{2}-\tau_{1}$, and $0<\rho<1$. Then, the time derivatives of $V(t)$ along the trajectories of the dynamic systems (13) satisfy

$$
\begin{aligned}
\dot{V}_{1}(t)= & x^{T}(t)\left(P^{T} A(t)+A^{T}(t) P\right) x(t) \\
& +2 x^{T}(t) P^{T} A_{\tau}(t) x(t-\tau(t)), \\
\dot{V}_{2}(t)= & \varepsilon x^{T}(t) Q_{1} x(t) \\
& -\varepsilon(1-\dot{\tau}(t)) x^{T}(t-\tau(t)) Q_{1} x(t-\tau(t)) \\
& +x^{T}(t) Q_{2} x(t)-x^{T}\left(t-\tau_{1}\right) Q_{2} x\left(t-\tau_{1}\right) \\
& +x^{T}\left(t-\tau_{1}\right) S_{1} x\left(t-\tau_{1}\right) \\
& -x^{T}\left(t-\tau_{\rho}\right) S_{1} x\left(t-\tau_{\rho}\right) \\
& +x^{T}\left(t-\tau_{\rho}\right) S_{2} x\left(t-\tau_{\rho}\right) \\
& -x^{T}\left(t-\tau_{2}\right) S_{2} x\left(t-\tau_{2}\right), \\
\dot{V}_{3}(t)= & \left(\tau_{2}-\tau_{1}\right)(E \dot{x}(t))^{T} R(E \dot{x}(t)) \\
& -\int_{t-\tau_{2}}^{t-\tau_{1}} \dot{x}^{T}(s) E^{T} R E \dot{x}(s) d s
\end{aligned}
$$




$$
\begin{gathered}
=\left(\tau_{2}-\tau_{1}\right)(E \dot{x}(t))^{T} R(E \dot{x}(t)) \\
-\int_{t-\tau_{\rho}}^{t-\tau_{1}} \dot{x}^{T}(s) E^{T} R E \dot{x}(s) d s \\
\quad-\int_{t-\tau_{2}}^{t-\tau_{\rho}} \dot{x}^{T}(s) E^{T} R E \dot{x}(s) d s .
\end{gathered}
$$

Denoting $\Gamma_{1}=\left[\begin{array}{lllll}A(t) & 0 & 0 & 0 & A_{\tau}(t)\end{array}\right], \zeta_{1}^{T}(t)=$ $\left[\begin{array}{lllll}x^{T}(t) & x^{T}\left(t-\tau_{1}\right) & x^{T}\left(t-\tau_{\rho}\right) & x^{T}\left(t-\tau_{2}\right) & x^{T}(t-\tau(t))\end{array}\right]$ and $\beta_{1}^{T}(t, s)=\left[\zeta_{1}^{T}(t)(E \dot{x}(s))^{T}\right]$, and from Newton-Leibniz formula, we can easily obtain that

$$
\begin{aligned}
& -2 \zeta_{1}^{T}(t) V^{T}\left[E x\left(t-\tau_{1}\right)-E x\left(t-\tau_{\rho}\right)\right. \\
& \left.-\int_{t-\tau_{\rho}}^{t-\tau_{1}} E \dot{x}(s) d s\right]=0, \\
& -2 \zeta_{1}^{T}(t) W^{T}\left[E x\left(t-\tau_{\rho}\right)-E x\left(t-\tau_{2}\right)\right. \\
& \left.-\int_{t-\tau_{2}}^{t-\tau_{\rho}} E \dot{x}(s) d s\right]=0 .
\end{aligned}
$$

Therefore, a straightforward computation gives

$$
\begin{aligned}
& \dot{V}(t)=x^{T}(t)\left[P^{T} A(t)+A^{T}(t) P+\varepsilon Q_{1}+Q_{2}\right] x(t) \\
& +2 x^{T}(t) P^{T} A_{\tau}(t) x(t-\tau(t))-\varepsilon(1-\dot{\tau}(t)) x^{T}(t \\
& -\tau(t)) Q_{1} x(t-\tau(t))+x^{T}\left(t-\tau_{1}\right)\left(S_{1}-Q_{2}\right) x(t \\
& \left.-\tau_{1}\right)+x^{T}\left(t-\tau_{\rho}\right)\left(S_{2}-S_{1}\right) x\left(t-\tau_{\rho}\right)+x^{T}(t \\
& \left.-\tau_{2}\right)\left(-S_{2}\right) x\left(t-\tau_{2}\right)+\left(\tau_{2}-\tau_{1}\right)(E \dot{x}(t))^{T} R(E \dot{x}(t)) \\
& -\int_{t-\tau_{\rho}}^{t-\tau_{1}} \dot{x}^{T}(s) E^{T} R E \dot{x}(s) d s-\int_{t-\tau_{2}}^{t-\tau_{\rho}} \dot{x}^{T}(s) \\
& \cdot E^{T} R E \dot{x}(s) d s+\int_{t-\tau_{\rho}}^{t-\tau_{1}} 2 \zeta_{1}^{T}(t) V^{T} E \dot{x}(s) d s \\
& +\int_{t-\tau_{2}}^{t-\tau_{\rho}} 2 \zeta_{1}^{T}(t) W^{T} E \dot{x}(s) d s-2 \zeta_{1}^{T}(t) \\
& \cdot V^{T} E\left[\begin{array}{lllll}
0 & I & -I & 0 & 0
\end{array}\right] \zeta_{1}(t)-2 \zeta_{1}^{T}(t) \\
& \text { - } W^{T} E\left[\begin{array}{lllll}
0 & 0 & I & -I & 0
\end{array}\right] \zeta_{1}(t) \leq \frac{1}{\tau_{2}-\tau_{1}} \\
& \cdot \int_{t-\tau_{\rho}}^{t-\tau_{1}}\left[\zeta_{1}^{T}(t) \Psi(t) \zeta_{1}(t)\right. \\
& +2\left(\tau_{2}-\tau_{1}\right) \zeta_{1}^{T}(t) V^{T} E \dot{x}(s)
\end{aligned}
$$

$$
\begin{aligned}
& \left.-\left(\tau_{2}-\tau_{1}\right) \dot{x}^{T}(s) E^{T} R E \dot{x}(s)\right] d s+\frac{1}{\tau_{2}-\tau_{1}} \\
& \cdot \int_{t-\tau_{2}}^{t-\tau_{\rho}}\left[\zeta_{1}^{T}(t) \Psi(t) \zeta_{1}(t)\right. \\
& +2\left(\tau_{2}-\tau_{1}\right) \zeta_{1}^{T}(t) W^{T} E \dot{x}(s) \\
& \left.-\left(\tau_{2}-\tau_{1}\right) \dot{x}^{T}(s) E^{T} R E \dot{x}(s)\right] d s=\frac{1}{\left(\tau_{2}-\tau_{1}\right)} \\
& \cdot \int_{t-\tau_{\rho}}^{t-\tau_{1}} \beta_{1}^{T}(t, s)\left[\begin{array}{c}
\Psi(t) \\
*
\end{array}-\left(\tau_{2}-\tau_{1}\right) V^{T}\right] \tau_{1}(t, s) d s \\
& +\frac{1}{\left(\tau_{2}-\tau_{1}\right)} \int_{t-\tau_{2}}^{t-\tau_{\rho}} \beta_{1}^{T}(t, s) \\
& \cdot\left[\begin{array}{c}
\Psi(t)\left(\tau_{2}-\tau_{1}\right) W^{T} \\
* \quad-\left(\tau_{2}-\tau_{1}\right) R
\end{array}\right] \beta_{1}(t, s) d s .
\end{aligned}
$$

Then, if conditions (15)-(16) hold, there exists $\alpha>0$ such that $\dot{V}\left(x_{t}\right)<\alpha\left\|x_{t}\right\|$. By Lemma 3, we conclude that the unforced fuzzy singular system (13) is stable. This completes the proof.

Remark 8. In some existing literature, for example, [22, 23], some delay-dependent criteria are given in terms of LMIs to guarantee that the fuzzy singular system is admissible by using LKF approach and integral inequality, such as Lemma 2 in [22] and Lemma 2.3 in [23]. However, in the proof of our result of Theorem 7 , we use one identical equality to estimate the upper bound of the derivative of $V(t)$ without any model transformation. Moreover, it is interesting to mention that this study presents criteria based on the free weighting matrix method, in which the bounding techniques on some cross product terms are not involved [11,23]. The major feature of this method is to reduce the conservatism engendered by the system transformations and the bounding techniques.

Remark 9. For time delay systems, the Lyapunov functional candidate always involves the integral term $\int_{-\tau}^{0} \int_{t+\theta}^{t} \dot{x}^{T}(s) R \dot{x}(s) d s d \theta$, and the derivative of it was estimated as $\tau \dot{x}^{T}(t) R \dot{x}(t)-\int_{t-\tau}^{t} \dot{x}^{T}(s) R \dot{x}(s) d s$. However, the term $\int_{t-\tau}^{t} \dot{x}^{T}(s) R \dot{x}(s) d s$ was ignored [25-27], or some useful negative integral term was lost; see, for example, [28, 29]. Instead, in this paper all those terms $\int_{t-\tau_{\rho}}^{t-\tau_{1}} \dot{x}^{T}(s) R \dot{x}(s) d s$ and $\int_{t-\tau_{2}}^{t-\tau_{\rho}} \dot{x}^{T}(s) R \dot{x}(s) d s$, which contain a great amount of useful information about systems, are preserved. Therefore, it is obvious to see that this method will lead to less conservatives than the existing ones in [25-29]. Furthermore, the introduction of parameter $\varepsilon(\varepsilon \geq 0)$ indicates that Lemma 3 can be suitable for time-varying delay $\tau(t)$ being unknown or not differentiable; that is, in the case of time-varying delay $\tau(t)$ not differentiable, one can set $\varepsilon=0$. 
In order to estimate system faults, the following fault estimation observer is constructed:

$$
\begin{aligned}
E \dot{\bar{x}}(t)= & A(t) \hat{x}(t)+A_{\tau}(t) \hat{x}(t-\tau(t))+B(t) u(t) \\
& +B_{f}(t) \widehat{f}(t)-L(t)(\widehat{y}(t)-y(t)), \\
\widehat{y}(t)= & C(t) \hat{x}(t)+C_{\tau}(t) \hat{x}(t-\tau(t))+D(t) u(t) \\
& +D_{f}(t) \hat{f}(t), \\
\dot{\hat{f}}(t)= & -F(t)(\hat{y}(t)-y(t)),
\end{aligned}
$$

where $\widehat{x}(t) \in \mathbb{R}^{n}$ is the observer state, $\widehat{y}(t) \in \mathbb{R}^{l}$ is the observer output, and $\widehat{f}(t) \in \mathbb{R}^{q}$ is an estimate of fault $f(t)$. The objective is to design the appropriate dimension gain matrices $L(t) \in \mathbb{R}^{n \times l}$ and $F(t) \in \mathbb{R}^{q \times l}$ and estimate the fault despite the presence of the disturbance and state delay, where $L(t)=\sum_{i=1}^{r} \mu_{i}(\xi(t)) L_{i}$ and $F(t)=\sum_{i=1}^{r} \mu_{i}(\xi(t)) F_{i}$. Let us define $e_{x}(t)=\widehat{x}(t)-x(t), e_{y}(t)=\widehat{y}(t)-y(t), e_{f}(t)=\widehat{f}(t)-f(t)$, and $e^{T}(t)=\left[e_{x}^{T}(t), e_{f}^{T}(t)\right], \omega^{T}(t)=\left[d^{T}(t), \dot{f}^{T}(t)\right]$; then the error dynamic systems are deduced from (7) and (36) as follows:

$$
\begin{aligned}
\bar{E} \dot{e}(t)= & {[\bar{A}(t)-\bar{L}(t) \bar{C}(t)] e(t) } \\
& +\left[\bar{A}_{\tau}(t)-\bar{L}(t) \bar{C}_{\tau}(t)\right] e(t-\tau(t)) \\
& +\left[\bar{L}(t) \bar{D}_{d}(t)-\bar{B}_{d}(t)\right] \omega(t), \\
e_{y}(t)= & \bar{C}(t) e(t)+\bar{C}_{\tau}(t) e(t-\tau(t))-\bar{D}_{d}(t) \omega(t),
\end{aligned}
$$

$$
\begin{aligned}
\zeta_{2}^{T}(t) & =\left[\begin{array}{llllll}
e^{T}(t) & e^{T}\left(\mathrm{t}-\tau_{1}\right) & e^{T}\left(t-\tau_{\rho}\right) & e^{T}\left(t-\tau_{2}\right) & e^{T}(t-\tau(t)) & \omega^{T}(t)
\end{array}\right] \\
\Gamma_{2} & =\left[\begin{array}{lllllll}
\bar{A}(t)-\bar{L}(t) \bar{C}(t) & 0 & 0 & 0 & \bar{A}_{\tau}(t)-\bar{L}(t) \bar{C}_{\tau}(t) & \bar{L}(t) \bar{D}_{d}(t)-\bar{B}_{d}(t)
\end{array}\right]
\end{aligned}
$$

where

$$
\begin{aligned}
& \bar{E}=\left[\begin{array}{ll}
E & 0 \\
0 & I_{q}
\end{array}\right], \\
& \bar{A}(t)=\left[\begin{array}{cc}
A(t) & B_{f}(t) \\
0 & 0
\end{array}\right], \\
& \bar{A}_{\tau}(t)=\left[\begin{array}{cc}
A_{\tau}(t) & 0 \\
0 & 0
\end{array}\right] \text {, } \\
& \bar{B}_{d}(t)=\left[\begin{array}{cc}
B_{d}(t) & 0 \\
0 & I_{q}
\end{array}\right] \text {, } \\
& \bar{L}(t)=\left[\begin{array}{l}
L(t) \\
F(t)
\end{array}\right], \\
& \bar{C}(t)=\left[\begin{array}{ll}
C(t) & D_{f}(t)
\end{array}\right], \\
& \bar{C}_{\tau}(t)=\left[\begin{array}{ll}
C_{\tau}(t) & 0
\end{array}\right], \\
& \bar{D}_{d}(t)=\left[\begin{array}{ll}
D_{d}(t) & 0
\end{array}\right] .
\end{aligned}
$$

Therefore, $H_{\infty}$ robust fault estimation observer design problem to be addressed in this paper can be formulated as follows: (i) The error dynamic system (37) with $\omega(t)=0$ is admissible for any time delay satisfying (2); (ii) for a given scalar $\gamma$, the following $H_{\infty}$ performance is satisfied:

$$
\int_{0}^{L}\left\|e_{f}(t)\right\|^{2} d t \leq \gamma^{2} \int_{0}^{L}\|\omega(t)\|^{2} d t
$$

for all $L>0$ and $\omega(t) \in L_{2}[0, \infty)$ under zero initial conditions.

For simplicity, we introduce the following vectors:
Then, the state of error dynamics (37) can be rewritten as $\bar{E} \dot{e}(t)=\Gamma_{2} \zeta_{2}(t)$.

Remark 10. From error dynamics (37), we can see that the new matrices $\bar{A}(t), \bar{A}_{\tau}(t), \bar{C}(t), \bar{C}_{\tau}(t), \bar{B}_{d}(t)$, and $\bar{D}_{d}(t)$ are known matrices, while the matrices $\bar{L}(t)$ contain two matrices $L(t)$ and $F(t)$ that have to be designed. Therefore, the proposed robust fault estimation observer design is converted to the problem of seeking the gain matrix $\bar{L}(t)$.

Next, a fuzzy augmented fault estimation observer design method under $H_{\infty}$ performance is proposed to achieve robust fault estimation by following lemma under timevarying state delay.
Lemma 11. For the given positive scalars $\tau_{1}, \tau_{2}, \tau_{D}$, and $\gamma$, the error dynamic system (37) is admissible with $\omega(t)=$ 0 while satisfying a prescribed $H_{\infty}$ performance (39), if there exist appropriately dimensional matrices $P, Q_{1}>0$, $Q_{2}>0, S_{1}>0, S_{2}>0, R>0$, and $\bar{L}(t)$ and free weighting matrices $V=\left[\begin{array}{llllll}V_{1} & V_{2} & V_{3} & V_{4} & V_{5} & V_{6}\end{array}\right]$ and $W=\left[\begin{array}{llllll}W_{1} & W_{2} & W_{3} & W_{4} & W_{5} & W_{6}\end{array}\right]$, such that the following inequalities hold:

$$
\begin{aligned}
\bar{E}^{T} P & =P^{T} \bar{E} \geq 0, \\
\Delta_{1}(t) & =\left[\begin{array}{cc}
\Phi(t) & \left(\tau_{2}-\tau_{1}\right) V^{T} \\
* & -\left(\tau_{2}-\tau_{1}\right) R
\end{array}\right]<0, \\
\Delta_{2}(t) & =\left[\begin{array}{cc}
\Phi(t) & \left(\tau_{2}-\tau_{1}\right) W^{T} \\
* & -\left(\tau_{2}-\tau_{1}\right) R
\end{array}\right]<0,
\end{aligned}
$$


where

$$
\begin{aligned}
\Phi(t) & =\Phi_{1}(t)+\left(\tau_{2}-\tau_{1}\right) \Gamma_{2}^{T} R \Gamma_{2}, \\
\Phi_{1}(t) & =\left[\begin{array}{cccccc}
\Phi_{11}(t) & -V_{1}^{T} E & V_{1}^{T} E-W_{1}^{T} E & W_{1}^{T} E & \Phi_{15}(t) & \Phi_{16}(t) \\
* & \Phi_{22} & \Phi_{23} & W_{2}^{T} E-E^{T} V_{4} & -E^{T} V_{5} & -E^{T} V_{6} \\
* & * & \Phi_{33} & \Phi_{34} & E^{T} V_{5}-E^{T} W_{5} & E^{T} V_{6}-E^{T} W_{6} \\
* & * & * & \Phi_{44} & E^{T} W_{5} & E^{T} W_{6} \\
* & * & * & * & -\varepsilon\left(1-\tau_{D}\right) Q_{1} & 0 \\
* & * & * & * & * & -\gamma^{2} I
\end{array}\right]
\end{aligned}
$$

with

$$
\begin{aligned}
\Phi_{11}(t)= & \operatorname{sym}\left(P^{T}(\bar{A}(t)-\bar{L}(t) \bar{C}(t))\right)+\varepsilon Q_{1}+Q_{2} \\
& +\bar{I}_{q} \bar{I}_{q}^{T}, \\
\Phi_{15}(t)= & P^{T}\left(\bar{A}_{\tau}(t)-\bar{L}(t) \bar{C}_{\tau}(t)\right), \\
\Phi_{16}(t)= & P^{T}\left(\bar{L}_{(}(t) \bar{D}_{d}(t)-\bar{B}_{d}(t)\right), \\
\bar{I}_{q}^{T}= & {\left[\begin{array}{ll}
0 & I_{q}
\end{array}\right], } \\
\Phi_{22}= & S_{1}-Q_{2}-V_{2}^{T} E-E^{T} V_{2}, \\
\Psi_{23}= & V_{2}^{T} E-W_{2}^{T} E-E^{T} V_{3}, \\
\Psi_{33}= & S_{2}-S_{1}+\operatorname{sym}\left(V_{3}^{T} E-W_{3}^{T} E\right), \\
\Psi_{34}= & W_{3}^{T} E-E^{T} V_{4}-E^{T} W_{4}, \\
\Psi_{44}= & -S_{2}+W_{4}^{T} E+E^{T} W_{4} .
\end{aligned}
$$

Proof. First, we show that the error dynamic system (37) with $\omega(t)=0$ is regular and impulse-free. Since $\bar{E}$ is singular and $\operatorname{rank}(\bar{E})=g+q$, there always exist two nonsingular matrices $\widehat{G} \in \mathbb{R}^{(n+q) \times(n+q)}$ and $\widehat{U} \in \mathbb{R}^{(n+q) \times(n+q)}$ such that

$$
\widehat{G} \bar{E} \widehat{H}=\left[\begin{array}{cc}
I_{g+q} & 0 \\
0 & 0
\end{array}\right] \text {. }
$$

Accordingly, denote

$$
\begin{gathered}
\widehat{G}(\bar{A}(t)-\bar{L}(t) \bar{C}(t)) \widehat{H}=\widehat{G} \widehat{A}(t) \widehat{H} \\
=\left[\begin{array}{ll}
\widehat{A}_{11}(t) & \widehat{A}_{12}(t) \\
\widehat{A}_{21}(t) & \widehat{A}_{22}(t)
\end{array}\right], \\
\widehat{G}^{-T} P \widehat{H}=\left[\begin{array}{ll}
\widehat{P}_{11} & \widehat{P}_{12} \\
\widehat{P}_{21} & \widehat{P}_{22}
\end{array}\right] .
\end{gathered}
$$

From (41) and using the expressions in (46)-(47), it is easy to obtain that $\widehat{P}_{12}=0$. Since (42)-(43) hold, we have $\Phi(t)=$
$\Phi_{1}(t)+\left(\tau_{2}-\tau_{1}\right) \Gamma_{2}^{T} R \Gamma_{2}<0$. Moreover, noting $R>0$ and by Schur complement, we can get $\Phi_{11}(t)=\operatorname{sym}\left(P^{T}(\bar{A}(t)-\right.$ $\bar{L}(t) \bar{C}(t)))+\varepsilon Q_{1}+Q_{2}+\bar{I}_{q} \bar{I}_{q}^{T}<0$. Then, premultiplying and postmultiplying $\Phi_{11}(t)<0$ by $\widehat{H}^{T}$ and $\widehat{H}$, respectively, we have $\widehat{A}_{22}^{T}(t) \widehat{P}_{22}+\widehat{P}_{22}^{T} \widehat{A}_{22}(t)<0$, which implies that $\widehat{A}_{22}(t)$ is nonsingular, and thus the pair $(\bar{E}, \widehat{A}(t))$ is regular and impulse-free. Hence, the error dynamic system (37) is regular and impulse-free for any time delay $\tau(t)$ satisfying (2) when inequalities (41)-(43) hold. Next, we will prove that the error dynamic system (37) is stable with $H_{\infty}$ performance. To this end, the Lyapunov-Krasovskii functional candidate is constructed as follows:

$$
\begin{aligned}
V(t)= & e^{T}(t) \bar{E}^{T} P e(t)+\varepsilon \int_{t-\tau(t)}^{t} e^{T}(s) Q_{1} e(s) d s \\
& +\int_{t-\tau_{1}}^{t} e^{T}(s) Q_{2} e(s) d s \\
& +\int_{t-\tau_{\rho}}^{t-\tau_{1}} e^{T}(s) S_{1} e(s) d s \\
& +\int_{t-\tau_{2}}^{t-\tau_{\rho}} e^{T}(s) S_{2} e(s) d s \\
& +\int_{-\tau_{2}}^{-\tau_{1}} \int_{t+\theta}^{t} \dot{e}^{T}(s) \bar{E}^{T} R \bar{E} \dot{e}(s) d s d \theta,
\end{aligned}
$$

where the unknown matrices $P, Q_{1}>0, Q_{2}>0, S_{1}>0, S_{2}>$ 0 , and $R>0$ are to be determined. Then, the time derivatives of $V(t)$ along the trajectories of the error dynamic system (37) satisfy

$$
\begin{aligned}
\dot{V} & (t)=e^{T}(t)\left[P^{T}(\bar{A}(t)-\bar{L}(t) \bar{C}(t))\right. \\
& \left.+(\bar{A}(t)-\bar{L}(t) \bar{C}(t))^{T} P+\varepsilon Q_{1}+Q_{2}\right] e(t) \\
& +2 e^{T}(t) P^{T}\left(\bar{A}_{\tau}(t)-\bar{L}(t) \bar{C}_{\tau}(t)\right) e(t-\tau(t)) \\
& +2 e^{T}(t) P^{T}\left(\bar{L}(t) \bar{D}_{d}(t)-\bar{B}_{d}(t)\right) \omega(t)-\varepsilon(1
\end{aligned}
$$




$$
\begin{aligned}
& -\dot{\tau}(t)) e^{T}(t-\tau(t)) Q_{1} e(t-\tau(t))+e^{T}\left(t-\tau_{1}\right)\left(S_{1}\right. \\
& \left.-Q_{2}\right) e\left(t-\tau_{1}\right)+e^{T}\left(t-\tau_{\rho}\right)\left(S_{2}-S_{1}\right) e\left(t-\tau_{\rho}\right) \\
& +e^{T}\left(t-\tau_{2}\right)\left(-S_{2}\right) e\left(t-\tau_{2}\right)+\left(\tau_{2}-\tau_{1}\right)(\bar{E} \dot{e}(t))^{T} \\
& \cdot R(\bar{E} \dot{e}(t))-\int_{t-\tau_{2}}^{t-\tau_{1}} \dot{e}^{T}(s) \bar{E}^{T} R \bar{E} \dot{e}(s) d s .
\end{aligned}
$$

Denoting $\beta_{2}^{T}(t, s)=\left[\zeta_{2}^{T}(t)(\bar{E} \dot{e}(s))^{T}\right]$, and from NewtonLeibniz formula, a straightforward computation gives

$$
\begin{aligned}
& \dot{V}(t)+e_{f}^{T}(t) e_{f}(t)-\gamma^{2} \omega^{T}(t) \omega(t)=\dot{V}(t)+e^{T}(t) \\
& \cdot \bar{I}_{q} \bar{I}_{q}^{T} e(t)-\gamma^{2} \omega^{T}(t) \omega(t)=e^{T}(t)\left[P^{T}(\bar{A}(t)\right. \\
& -\bar{L}(t) \bar{C}(t))+(\bar{A}(t)-\bar{L}(t) \bar{C}(t))^{T} P+\varepsilon Q_{1}+Q_{2} \\
& \left.+\bar{I}_{q} \bar{I}_{q}^{T}\right] e(t)+2 e^{T}(t) P^{T}\left(\bar{A}_{\tau}(t)-\bar{L}(t) \bar{C}_{\tau}(t)\right) e(t \\
& -\tau(t))+2 e^{T}(t) P^{T}\left(\bar{L}(t) \bar{D}_{d}(t)-\bar{B}_{d}(t)\right) \omega(t) \\
& -\varepsilon(1-\dot{\tau}(t)) e^{T}(t-\tau(t)) Q_{1} e(t-\tau(t))+e^{T}(t \\
& \left.-\tau_{1}\right)\left(S_{1}-Q_{2}\right) e\left(t-\tau_{1}\right)+e^{T}\left(t-\tau_{\rho}\right)\left(S_{2}-S_{1}\right) e(t \\
& \left.-\tau_{\rho}\right)+e^{T}\left(t-\tau_{2}\right)\left(-S_{2}\right) e\left(t-\tau_{2}\right)-\gamma^{2} \omega^{T}(t) \omega(t) \\
& +\left(\tau_{2}-\tau_{1}\right)(\bar{E} \dot{e}(t))^{T} R(\bar{E} \dot{e}(t))-\int_{t-\tau_{\rho}}^{t-\tau_{1}} \dot{e}^{T}(s) \\
& \cdot \bar{E}^{T} R \bar{E} \dot{e}(s) d s-\int_{t-\tau_{2}}^{t-\tau_{\rho}} \dot{e}^{T}(s) \bar{E}^{T} R \bar{E} \dot{e}(s) d s \\
& -2 \zeta_{2}^{T}(t) V^{T} \bar{E}\left[\begin{array}{llllll}
0 & I & -I & 0 & 0 & 0
\end{array}\right] \zeta_{2}(t) \\
& +\int_{t-\tau_{\rho}}^{t-\tau_{1}} 2 \zeta_{2}^{T}(t) V^{T} \bar{E} \dot{e}(s) d s-2 \zeta_{2}^{T}(t) \\
& \text { - } W^{T} \bar{E}\left[\begin{array}{llllll}
0 & 0 & I & -I & 0 & 0
\end{array}\right] \zeta_{2}(t)+\int_{t-\tau_{2}}^{t-\tau_{\rho}} 2 \zeta_{2}^{T}(t) \\
& \cdot W^{T} \bar{E} \dot{e}(s) d s \leq \frac{1}{\tau_{2}-\tau_{1}} \\
& \cdot \int_{t-\tau_{\rho}}^{t-\tau_{1}}\left[\zeta_{2}^{T}(t) \Phi(t) \zeta_{2}(t)\right. \\
& +2\left(\tau_{2}-\tau_{1}\right) \zeta_{2}^{T}(t) V^{T} \bar{E} \dot{e}(s) \\
& \left.-\left(\tau_{2}-\tau_{1}\right) \dot{e}^{T}(s) \bar{E}^{T} R \bar{E} \dot{e}(s)\right] d s+\frac{1}{\tau_{2}-\tau_{1}}
\end{aligned}
$$

$$
\begin{aligned}
& \cdot \int_{t-\tau_{2}}^{t-\tau_{\rho}}\left[\zeta_{2}^{T}(t) \Phi(t) \zeta_{2}(t)\right. \\
& +2\left(\tau_{2}-\tau_{1}\right) \zeta_{2}^{T}(t) W^{T} \bar{E} \dot{e}(s) \\
& \left.-\left(\tau_{2}-\tau_{1}\right) \dot{e}^{T}(s) \bar{E}^{T} R \bar{E} \dot{e}(s)\right] d s=\frac{1}{\tau_{2}-\tau_{1}} \\
& \cdot \int_{t-\tau_{\rho}}^{t-\tau_{1}} \beta_{2}^{T}(t, s)\left[\begin{array}{cc}
\Phi(t) & \left(\tau_{2}-\tau_{1}\right) V^{T} \\
* & -\left(\tau_{2}-\tau_{1}\right) R
\end{array}\right] \beta_{2}(t, s) d s \\
& +\frac{1}{\tau_{2}-\tau_{1}} \int_{t-\tau_{2}}^{t-\tau_{\rho}} \beta_{2}^{T}(t, s) \\
& \cdot\left[\begin{array}{cc}
\Phi(t) & \left(\tau_{2}-\tau_{1}\right) W^{T} \\
* & -\left(\tau_{2}-\tau_{1}\right) R
\end{array}\right] \beta_{2}(t, s) d s .
\end{aligned}
$$

If (42)-(43) hold, one has $\dot{V}(t)+e_{f}^{T}(t) e_{f}(t)-\gamma^{2} \omega^{T}(t) \omega(t)<0$. By noticing $V(L) \geq 0$ and $V(0)=0$ under zero initial conditions, we can conclude that (39) holds for all $L>0$ and any nonzero $\omega(t) \in L_{2}[0, \infty)$.

On the other hand, under conditions (42)-(43), by choosing the same Lyapunov function as (48) and following the similar line in the earlier deduction under conditions (42)-(43), then substitute $A_{i}$ and $A_{\tau i}$ by $(\bar{A}(t)-\bar{L}(t) \bar{C}(t))$ and $\left(\bar{A}_{\tau}(t)-\bar{L}(t) \bar{C}_{\tau}(t)\right)$; we can easily obtain that the time derivative of $V(t)$ along the solution of error dynamics (37) with $\omega(t)=0$ satisfies $\dot{V}\left(e_{t}\right)<\alpha\left\|e_{t}\right\|$ by using Theorem 7 , which indicates the stability of system (37). This complete the proof.

In the following, we will focus on the design of observer based on Lemma 11 and provide a new sufficient condition for the existence of robust fault estimation observer for fuzzy singular time delay system (3).

Theorem 12. For the given positive scalars $\tau_{1}, \tau_{2}, \tau_{D}, \delta$, and $\gamma$, the error dynamic system (37) is admissible with $\omega(t)=0$ while satisfying a prescribed $H_{\infty}$ performance (39), if there exist appropriately dimensional matrices $P, Q_{1}>$ $0, Q_{2}>0, S_{1}>0, S_{2}>0, R>0$, and $Y_{i}$ and free weighting matrices $V=\left[\begin{array}{llllll}V_{1} & V_{2} & V_{3} & V_{4} & V_{5} & V_{6}\end{array}\right]$ and $W=\left[\begin{array}{llllll}W_{1} & W_{2} & W_{3} & W_{4} & W_{5} & W_{6}\end{array}\right]$, such that the following inequalities hold:

$$
\begin{array}{rl}
\bar{E}^{T} P=P^{T} \bar{E} \geq 0, & \\
\Xi_{i i}<0 & i=1,2, \ldots, r, \\
\Xi_{i j}+\Xi_{j i} \leq 0 & 1 \leq i<j \leq r, \\
\Pi_{i i}<0 & i=1,2, \ldots, r, \\
\Pi_{i j}+\Pi_{j i} \leq 0 & 1 \leq i<j \leq r,
\end{array}
$$


where

$$
\Xi_{i j}=\left[\begin{array}{ccc}
\Phi_{i j} & \left(\tau_{2}-\tau_{1}\right) V^{T} & \sqrt{\left(\tau_{2}-\tau_{1}\right)} \bar{\Gamma}_{2 i j}^{T} \\
* & -\left(\tau_{2}-\tau_{1}\right) R & 0 \\
* & * & -2 \delta \bar{E}^{T} P+\delta^{2} R
\end{array}\right],
$$

$$
\Pi_{i j}=\left[\begin{array}{ccc}
\Phi_{i j} & \left(\tau_{2}-\tau_{1}\right) W^{T} & \sqrt{\left(\tau_{2}-\tau_{1}\right)} \bar{\Gamma}_{2 i j}^{T} \\
* & -\left(\tau_{2}-\tau_{1}\right) R & 0 \\
* & * & -2 \delta \bar{E}^{T} P+\delta^{2} R
\end{array}\right],
$$

where

$$
\Phi_{i j}=\left[\begin{array}{cccccc}
\Phi_{11 i j} & -V_{1}^{T} \bar{E} & V_{1}^{T} \bar{E}-W_{1}^{T} \bar{E} & W_{1}^{T} \bar{E} & \Phi_{15 i j} & \Phi_{16 i j} \\
* & \Phi_{22} & \Phi_{23} & W_{2}^{T} \bar{E}-\bar{E}^{T} V_{4} & -\bar{E}^{T} V_{5} & -\bar{E}^{T} V_{6} \\
* & * & \Phi_{33} & \Phi_{34} & \bar{E}^{T} V_{5}-\bar{E}^{T} W_{5} & \bar{E}^{T} V_{6}-\bar{E}^{T} W_{6} \\
* & * & * & \Phi_{44} & \bar{E}^{T} W_{5} & \bar{E}^{T} W_{6} \\
* & * & * & * & -\varepsilon\left(1-\tau_{D}\right) Q_{1} & 0 \\
* & * & * & * & * & -\gamma^{2} I
\end{array}\right]
$$

with

$$
\begin{aligned}
& \Phi_{11 i j}=\operatorname{sym}\left(P^{T} \bar{A}_{i}-Y_{i} \bar{C}_{j}\right)+\varepsilon Q_{1}+Q_{2}+\bar{I}_{q} \bar{I}_{q}^{T}, \\
& \Phi_{15 i j}=P^{T} \bar{A}_{\tau i}-Y_{i} \bar{C}_{\tau j} \text {, } \\
& \Phi_{16 i j}=Y_{i} \bar{D}_{d j}-P^{T} \bar{B}_{d i} \text {, } \\
& \Phi_{22}=S_{1}-Q_{2}-V_{2}^{T} E-E^{T} V_{2}, \\
& \Psi_{23}=V_{2}^{T} E-W_{2}^{T} E-E^{T} V_{3} \text {, } \\
& \Psi_{33}=S_{2}-S_{1}+\operatorname{sym}\left(V_{3}^{T} E-W_{3}^{T} E\right), \\
& \Psi_{34}=W_{3}^{T} E-E^{T} V_{4}-E^{T} W_{4} \text {, } \\
& \Psi_{44}=-S_{2}+W_{4}^{T} E+E^{T} W_{4}, \\
& \bar{I}_{q}^{T}=\left[\begin{array}{ll}
0 & I_{q}
\end{array}\right] \text {, } \\
& \bar{\Gamma}_{2 i j} \\
& =\bar{E}\left[P^{T} \bar{A}_{i}-Y_{i} \bar{C}_{j} \quad 0 \quad 0 \quad 0 \quad P^{T} \bar{A}_{\tau i}-Y_{i} \bar{C}_{\tau j} \quad Y_{i} \bar{D}_{d j}-P^{T} \bar{B}_{d i}\right] .
\end{aligned}
$$

Then the observer gain matrices can be obtained as $\bar{L}_{i}=\left[\begin{array}{c}L_{i} \\ F_{i}\end{array}\right]=$ $P^{-T} Y_{i}$.

Proof. For any scalar $\delta$, it follows from the fact $(\delta R-$ $P) R^{-1}(\delta R-P) \geq 0$ that $-P R^{-1} P \leq-2 \delta P+\delta^{2} R$. By Schur complement theorem, we can conclude that (42)-(43) hold if the following inequalities hold:

$$
\left[\begin{array}{ccc}
\Phi_{1}(t) & \left(\tau_{2}-\tau_{1}\right) V^{T} & \sqrt{\left(\tau_{2}-\tau_{1}\right)} \Gamma_{2}^{T} P \bar{E}^{T} \\
* & -\left(\tau_{2}-\tau_{1}\right) R & 0 \\
* & * & -2 \delta \bar{E} P^{T}+\delta^{2} R
\end{array}\right]<0
$$

$$
\left[\begin{array}{ccc}
\Phi_{1}(t) & \left(\tau_{2}-\tau_{1}\right) W^{T} & \sqrt{\left(\tau_{2}-\tau_{1}\right)} \Gamma_{2}^{T} P \bar{E}^{T} \\
* & -\left(\tau_{2}-\tau_{1}\right) R & 0 \\
* & * & -2 \delta \bar{E} P^{T}+\delta^{2} R
\end{array}\right]<0,
$$

where $\Phi_{1}(t)$ is defined in Lemma 11. Then, if (52)-(55) hold and with the changes of variables as $Y_{i}=P^{T} L_{i}$, we have

$$
\begin{aligned}
\Delta_{1}(t)= & \sum_{i=1}^{r} \mu_{i}^{2}(\xi(t)) \Xi_{i i} \\
& +\sum_{i=1}^{r} \sum_{i<j}^{r} \mu_{i}(\xi(t)) \mu_{j}(\xi(t))\left(\Xi_{i j}+\Xi_{j i}\right)<0, \\
\Delta_{2}(t)= & \sum_{i=1}^{r} \mu_{i}^{2}(\xi(t)) \Pi_{i i} \\
& +\sum_{i=1}^{r} \sum_{i<j}^{r} \mu_{i}(\xi(t)) \mu_{j}(\xi(t))\left(\Pi_{i j}+\Pi_{j i}\right)<0
\end{aligned}
$$

which imply that the error dynamics (37) are stable with $\omega(t)=0$ while satisfying the prescribed $H_{\infty}$ performance (39) by Lemma 11. The proof is completed.

Remark 13. Theorem 12 provides a criterion for designing $H_{\infty}$ robust fault estimation observer of fuzzy singular time delay systems, which guarantees the stability of the resulting dynamic error system with $H_{\infty}$ performance $\gamma>0$. As the delay term $\tau(t)$ is not simply enlarged, the proposed conditions are less conservative. Moreover, the proposed method is not only able to better depict the size and shape of the actuator fault but also able to estimate the sensor faults simultaneously.

Remark 14. Note that conditions (52)-(55) are LMIs. This indicates that the conditions (52)-(55) can be included as 
an optimization variable problems, which can be exploited to reduce the attenuation level bound. Then, the minimum attenuation level of $H_{\infty}$ performance can be obtained by the mincx function of Matlab toolbox. From the practical point of view, it is interesting to find an estimation law, which minimizes the disturbance rejection level $\gamma$ for the error dynamic system. This can be done by solving a convex optimization problem P: $\min \vartheta$ subject to (51)-(55) with $\vartheta=\gamma^{2}$.

Remark 15. In dealing with time-varying faults, there may be a time delay between the fault estimation and the system fault. This phenomenon results from the influence of fault variation. Theoretically, the attenuation level $\gamma_{\min }$ can be minimized so that the fault estimation is insensitive to the fault variation. However, the cost is that the fault estimation becomes less robust to disturbance noise. Therefore, the attenuation level of $\gamma_{\min }$, the fault variation, and disturbance are a trade-off.

\section{Numerical Examples}

In this section, three examples are given to show the effectiveness of our results. All the numerical results are calculated via the Yalmip toolbox of Matlab.

Example 1. Consider a continuous fuzzy singular system composed of two rules and the following system matrices $[22,23]$ :

$$
\begin{aligned}
E & =\left[\begin{array}{llll}
1 & 0 & 0 & 0 \\
0 & 1 & 0 & 0 \\
0 & 0 & 1 & 0 \\
0 & 0 & 0 & 0
\end{array}\right], \\
A_{1} & =\left[\begin{array}{cccc}
-3 & 0 & 0 & 0.2 \\
0 & -4 & 0.1 & 0 \\
0 & 0 & -0.1 & 0 \\
0.1 & 0.1 & -0.2 & -0.2
\end{array}\right], \\
A_{2} & =\left[\begin{array}{cccc}
-2 & 0 & 0 & -0.2 \\
0 & -2.5 & -0.1 & 0 \\
0 & -0.2 & -0.3 & 0 \\
0.1 & 0.1 & -0.2 & -0.2
\end{array}\right], \\
A_{\tau 2} & {\left[\begin{array}{cccc}
-0.5 & 0 & 0 & 0 \\
0 & -1 & 0 & 0 \\
0 & 0.1 & -0.5 & 0 \\
0 & 0.1 & -0.2 & 0 \\
0 & 0 & 0 & 0
\end{array}\right], } \\
A_{\tau 1} & {\left[\begin{array}{cccc}
-0.5 & 0 & 0 & 0 \\
0 & -1 & 0 & 0 \\
0 & 0 & 0
\end{array}\right] }
\end{aligned}
$$

Assume that the delay $\tau(t)$ satisfies (2) and set $\rho=0.5$. The obtained results are listed in Table 1 , where $N(m)$ stands for the total number of decision variables. Table 1 tabulates a comparison of the maximum allowable upper delay bound $\tau_{2}$ for a prescribed $\tau_{D}$. It can be seen from the table that our results are marked better than those obtained by the method in [22, 23]. Moreover, it is worth mentioning that the method proposed in this paper uses fewer number of LMIs scalar variables and fewer number of LMIs for stability computation; thus our method is more computationally efficient for improving the upper bound of delay; the stability criterion we derived is less conservative than those reported in the aforementioned papers.

Example 2. Consider the following singular time delay system:

$$
\begin{aligned}
{\left[\begin{array}{ll}
1 & 0 \\
0 & 0
\end{array}\right] \dot{x}(t)=} & {\left[\begin{array}{cc}
0.6341 & 0.5413 \\
-0.6121 & -1.1210
\end{array}\right] x(t) } \\
& +\left[\begin{array}{cc}
-0.4500 & 0 \\
0 & -0.1210
\end{array}\right] x(t-h) .
\end{aligned}
$$

To compare with the existing results, we assume that $\tau_{D}=0$ and $\rho=0.5$. Table 2 lists the comparison results on the maximum allowed time delay $\tau_{2}$ via the methods in $[10,20$, 22-24] and Theorem 7 in this paper. From the comparison result we can see that the stability criterion we derived by using free weighting matrix approach in this work is less conservative than those reported in [10, 20, 22-24].

Example 3. Consider the following fuzzy singular system with time-varying delay:

$$
\begin{aligned}
& E \dot{x}(t)=\sum_{i=1}^{2} \mu_{i}(\xi(t))\left\{A_{i} x(t)+A_{\tau i} x(t-\tau(t))\right. \\
& \left.+B_{i} u(t)+B_{f i} f(t)+B_{d i} d(t)\right\}, \\
& y(t)=\sum_{i=1}^{2} \mu_{i}(\xi(t))\left\{C_{i} x(t)+C_{\tau i} x(t-\tau(t))\right. \\
& \left.+D_{f i} f(t)+D_{d i} d(t)\right\},
\end{aligned}
$$

where

$$
\begin{aligned}
E & =\left[\begin{array}{lll}
1 & 0 & 0 \\
0 & 1 & 0 \\
0 & 0 & 0
\end{array}\right], \\
A_{1} & =\left[\begin{array}{ccc}
-0.60 & 1 & -1 \\
-0.65 & -0.5 & 0.2 \\
0.74 & -4 & -1
\end{array}\right], \\
A_{2} & =\left[\begin{array}{ccc}
-0.60 & 1 & -0.8 \\
-0.65 & -0.50 & 0.4 \\
0.82 & -6.37 & -1
\end{array}\right],
\end{aligned}
$$


TABLE 1: Allowable upper bound of $\tau_{2}$ for various $\tau_{D}$ in Example 1.

\begin{tabular}{lccccccc}
\hline$\tau_{D}$ & 0.1 & 0.35 & 0.6 & 0.85 & 0.9 & 0.95 & $N(m)$ \\
\hline Theorem 1 in [22] & 3.3623 & 2.9810 & 2.6010 & 1.8330 & 1.3080 & - & 24 \\
Theorem 3.1 in [23] & 3.3685 & 3.1560 & 3.1510 & 3.0760 & 2.6750 & 2.0780 & 19 \\
Theorem 7 & 4.1230 & 3.9891 & 3.7104 & 3.5247 & 3.4450 & 3.3702 \\
\hline
\end{tabular}

TABLE 2: Comparison of maximum allowed delays $\tau_{2}$ in Example 2.

\begin{tabular}{cccccc}
\hline$[10]$ & {$[20]$} & {$[24]$} & {$[22]$} & {$[23]$} & Theorem 7 \\
\hline- & 2.1328 & 2.1372 & 2.1372 & 2.3393 & 3.1324 \\
\hline
\end{tabular}

TABLE 3: Minimum index $\gamma$ for various $\delta$ in Example 3 with $\tau_{D}=0.2$.

\begin{tabular}{cccccc}
\hline$\delta$ & 0.5 & 1 & 2 & 5 & 10 \\
\hline$\tau=0.5$ & 1.6322 & 1.4559 & 1.3732 & 1.3465 & 1.5532 \\
\hline
\end{tabular}

$$
\begin{aligned}
& A_{\tau 1}=\left[\begin{array}{ccc}
0.07 & 0.02 & -0.01 \\
-0.09 & 0 & 0.02 \\
0.07 & 0.05 & 0
\end{array}\right], \\
& A_{\tau 2}=\left[\begin{array}{ccc}
0.07 & 0.12 & -0.01 \\
-0.08 & 0 & 0.03 \\
0.12 & 0.04 & 0
\end{array}\right] \text {, } \\
& B_{i}=B_{f i}=\left[\begin{array}{lll}
0.7 & 0 & 0
\end{array}\right], \\
& D_{d i}=0.2 \text {, } \\
& (i=1,2) \text {, } \\
& B_{d 1}=B_{d 2}=\left[\begin{array}{lll}
0.1 & 0.1 & 0.1
\end{array}\right], \\
& C_{1}=C_{2}=\left[\begin{array}{lll}
-0.2 & 0.5 & -0.15
\end{array}\right], \\
& C_{t 1}=C_{t 2}=\left[\begin{array}{lll}
-0.02 & 0.05 & -0.15
\end{array}\right] \text {. }
\end{aligned}
$$

Here, we consider the case where $\delta=2$ and the time-varying delay is given as $\tau(t)=0.3+0.2 \sin (t)$, and a straightforward calculation gives $\tau_{1}=0.1, \tau_{2}=0.5$, and $\tau_{D}=0.2$. By solving the conditions in (51)-(55), we obtain that the achieved $\gamma_{\min }=1.3140$, and the feasible solution is (due to space consideration, we do not list all the matrices here)

$$
P=\left[\begin{array}{cccc}
10.2168 & 11.6131 & 0.0000 & -4.2840 \\
11.6131 & 32.7665 & 0.0000 & -2.8547 \\
0.0000 & 0.0000 & 1.4842 & 0.0000 \\
-4.2840 & -2.8547 & 0.0000 & 2.1990
\end{array}\right]
$$

$$
Y_{1}=\left[\begin{array}{c}
4.0957 \\
50.1306 \\
-7.9107 \\
-3.6154
\end{array}\right],
$$

$$
Y_{2}=\left[\begin{array}{c}
4.2943 \\
48.5194 \\
-10.5807 \\
-3.7476
\end{array}\right] \text {. }
$$

The associate fault estimation observer gains in (36) are

$$
\begin{aligned}
& L_{1}=\left[\begin{array}{c}
-20.3217 \\
5.7954 \\
-5.3299
\end{array}\right], \\
& F_{1}=-33.7106, \\
& L_{2}=\left[\begin{array}{c}
-19.8103 \\
5.6276 \\
-7.1289
\end{array}\right], \\
& F_{2}=-32.9922 .
\end{aligned}
$$

According to Theorem 12, we can consider different $\delta$ to find the minimum index $\gamma$ for the given $\tau_{D}=0.2$; see $[25,28]$ for more details. The corresponding results are summarized in Table 3.

In order to illustrate the performance of robust fault estimation observer in dealing with fuzzy singular systems with time delay, first, an abrupt fault is simulated. It is assumed that the abrupt fault $f(t)$ is created as

$$
f(t)= \begin{cases}0 & 0 \leq t<10 \\ -10 & 10 \leq t \leq 30\end{cases}
$$

For simulation purpose, we choose the membership functions for Rules 1 and 2 to be $\mu_{1}(\xi(t))=1 /\left(1+\exp \left(x_{1}(t)+0.5\right)\right)$ and $\mu_{2}(\xi(t))=1-\mu_{1}(\xi(t))$ with the initial state condition as $x(0)=\left[\begin{array}{lll}-1.571 & -1.356 & 1.279\end{array}\right]^{T}$ while the initial estimate is $\widehat{x}(0)=\left[\begin{array}{lll}0 & 0 & 0\end{array}\right]^{T}$. Meanwhile, it is assumed that $d(t)$ is band-limited white noise with power 0.1 and sampling time 0.01s. Then, the fault estimation result of the robust fault estimation observer is depicted in Figure 1. Therein, the fault is depicted by red dash-and-dot line, and the fault estimation 


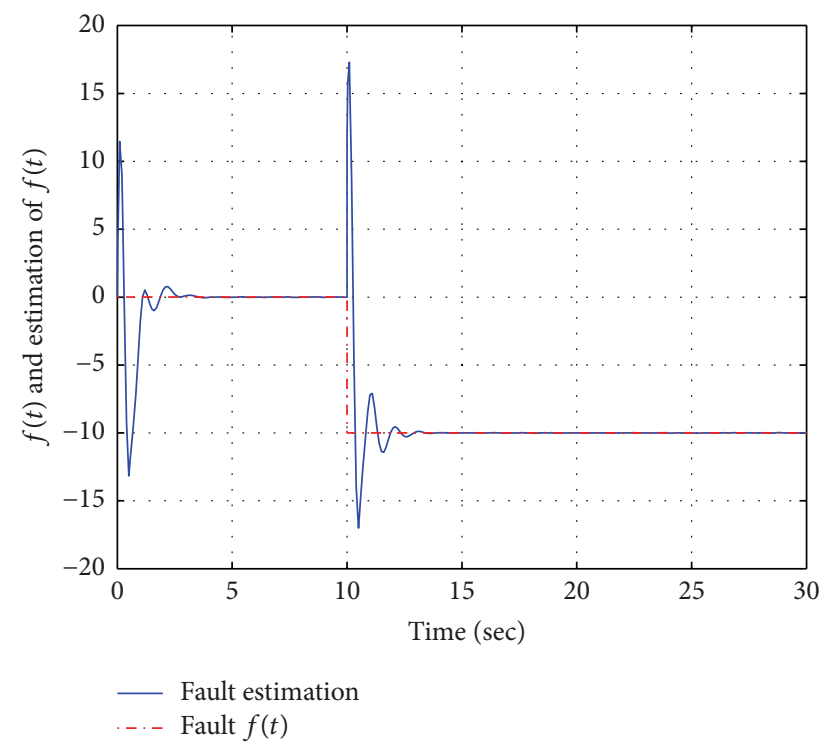

FIGURE 1: Fault estimation result of the robust fault estimation observer in abrupt fault case $f(t)$.

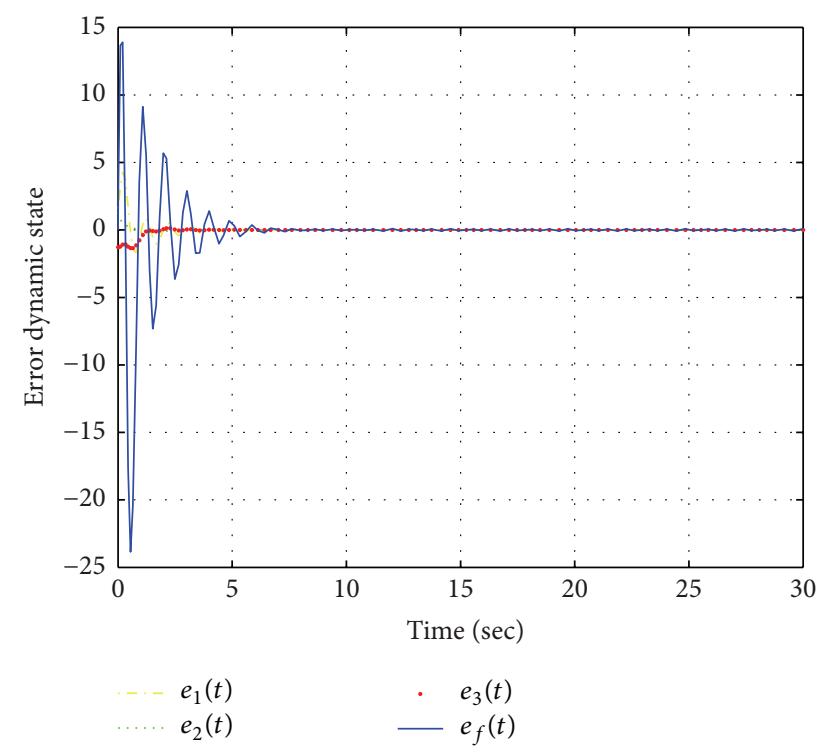

FIGURE 2: Response curves of error dynamics $e_{1}(t), e_{2}(t), e_{3}(t)$, and $e_{f}(t)$ in abrupt fault case $f(t)$.

is represented by the blue solid one. As shown in Figure 1, the robust fault estimation observer is insensitive to the model disturbance. Moreover, although there is estimation error, the fault estimate can quickly track the fault. This illustrates the fast convergence rate of the fault estimation observer in the face of initial estimation error. The simulation results shown in Figure 1 obviously illustrate that the proposed fault estimation has a good performance to estimate fault, and the error dynamic system is also stable in Figure 2. To illustrate the performance of robust fault estimation observer

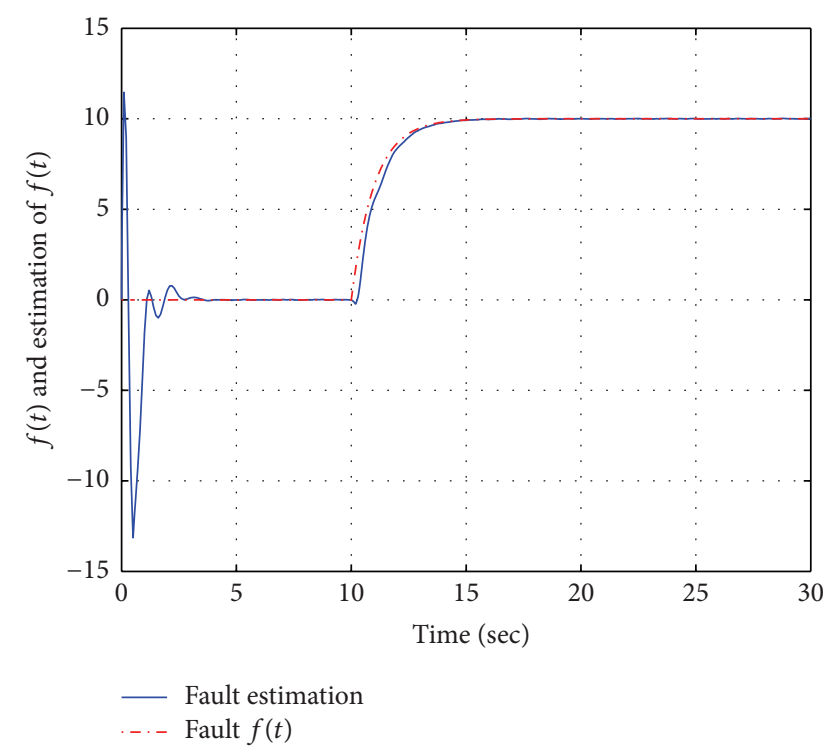

FIGURE 3: Fault estimation result of the robust fault estimation observer in fault $f_{1}(t)$.

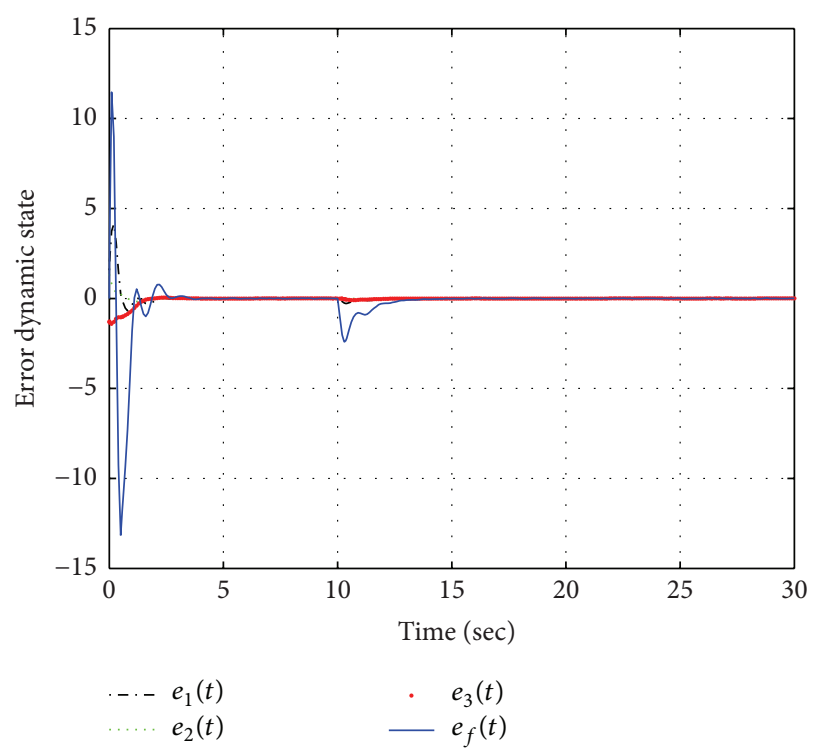

FIGURE 4: Response curves of error dynamics $e_{1}(t), e_{2}(t), e_{3}(t)$, and $e_{f}(t)$ in fault $f_{1}(t)$.

design method, the following time-varying fault is further considered:

$$
\begin{aligned}
& f_{1}(t)= \begin{cases}0 & 0 \leq t<10 \\
10\left(1-e^{-(t-5)}\right) & 10 \leq t \leq 20,\end{cases} \\
& f_{2}(t)= \begin{cases}0 & 0 \leq t<10 \\
0.5 \sin (0.8(t-5)) & 10 \leq t \leq 20 .\end{cases}
\end{aligned}
$$

In this situation, the fault estimation result is depicted in Figures 3 and 5. It can be seen from Figures 3 and 5 that the fault is estimated with satisfactory accuracy and 


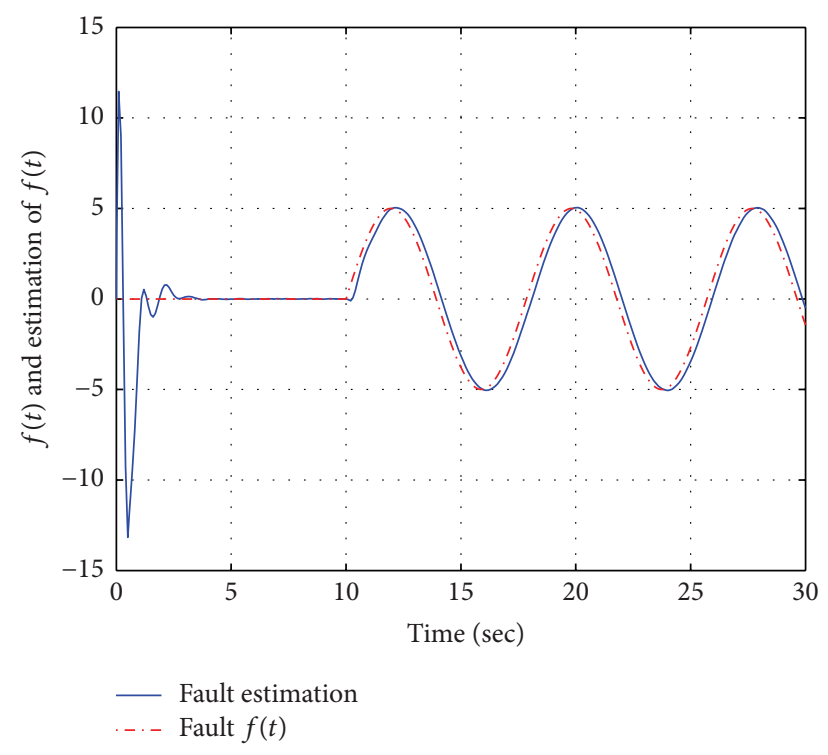

FIGURE 5: Fault estimation result of the robust fault estimation observer in fault $f_{2}(t)$.

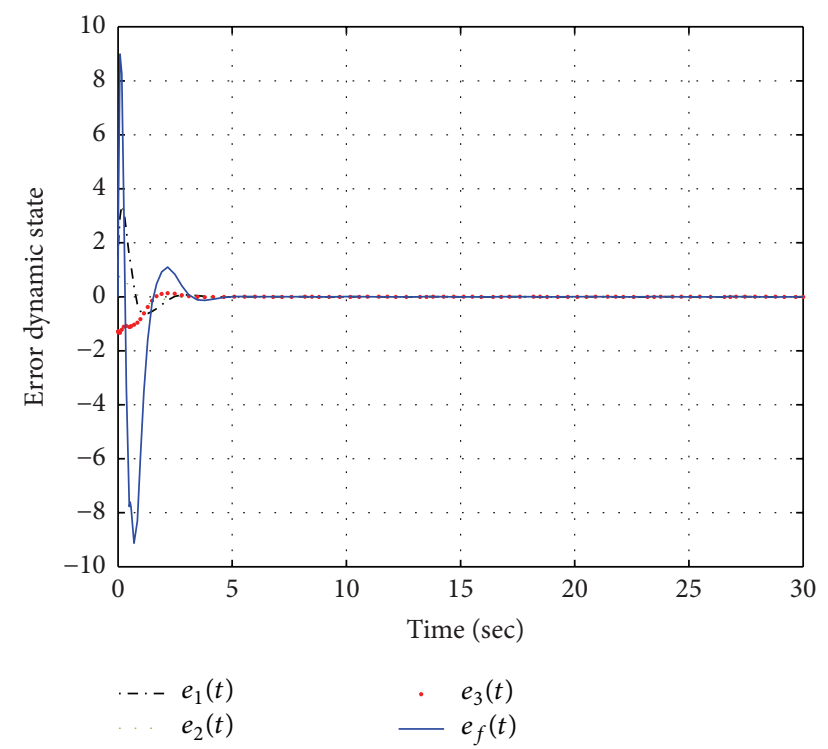

FIGURE 6: Response curves of error dynamics $e_{1}(t), e_{2}(t), e_{3}(t)$, and $e_{f}(t)$ in fault $f_{2}(t)$.

rapidity. The state of the error dynamic system is also stable in Figures 4 and 6. In [13], actuator fault estimation observer is designed for discrete-time linear parameter-varying descriptor systems, but the systems with time-varying delay case are not considered. In [9], pole assignment is used to ensure the fault estimation convergence speed while our method utilizes $H_{\infty}$ technique to attenuate the effect of fault variation. From Figures 3 and 5, it can be seen that these methods have similar fault convergence speed. Nevertheless, [9] only deals with regular systems not with time delay. Moreover, our method considers the fuzzy singular system with actuator and sensor faults simultaneously. Therefore, the proposed method is more general than that in $[9,13]$.

\section{Conclusion}

In this paper, the robust fault estimation problem for T-S fuzzy singular systems with time-varying delays is considered. By considering the fault as an auxiliary disturbance vector, based on the Lyapunov theorem and improved delay partitioning method with free weighting matrix approach, we give some less conservative criteria, which guarantee that the considered system is regular, impulse-free, and stable, while limiting the influence of disturbance despite the presence of actuator and sensor faults simultaneously. This paper proposes a novel fault estimation observer and presents an LMI-based design method for the fuzzy singular system. Finally, some numerical examples are used to demonstrate the effectiveness and performance of the proposed method.

\section{Conflict of Interests}

The authors declare that there is no conflict of interests regarding the publication of this paper.

\section{Acknowledgments}

This work is supported in part by the National Natural Science Foundation of China (Grant nos. 61533007, 61374146 and 61174215), Project 863 of China (Grant no. 2011AA060204), and IAPI Fundamental Research Funds (Grant no. 2013ZCX02-04).

\section{References}

[1] D. Theilliol, H. Noura, and J.-C. Ponsart, "Fault diagnosis and accommodation of a three-tank system based on analytical redundancy," ISA Transactions, vol. 41, no. 3, pp. 365-382, 2002.

[2] M. Rodrigues, D. Theilliol, M. Adam-Medina, and D. Sauter, "A fault detection and isolation scheme for industrial systems based on multiple operating models," Control Engineering Practice, vol. 16, no. 2, pp. 225-239, 2008.

[3] J. Bokor and Z. Szabó, "Fault detection and isolation in nonlinear systems," Annual Reviews in Control, vol. 33, no. 2, pp. 113-123, 2009.

[4] P. Mhaskar, C. McFall, A. Gani, P. D. Christofides, and J. F. Davis, "Isolation and handling of actuator faults in nonlinear systems," Automatica, vol. 44, no. 1, pp. 53-62, 2008.

[5] M. Liu, X. Cao, and P. Shi, "Fault estimation and tolerant control for fuzzy stochastic systems," IEEE Transactions on Fuzzy Systems, vol. 21, no. 2, pp. 221-229, 2013.

[6] Y. Zhang and J. Jiang, "Bibliographical review on reconfigurable fault-tolerant control systems," Annual Reviews in Control, vol. 32, no. 2, pp. 229-252, 2008.

[7] G. R. Duan, D. Howe, and R. J. Patton, "Robust fault detection in descriptor linear systems via generalized unknown input observers," International Journal of Systems Science, vol. 33, no. 5, pp. 369-377, 2002.

[8] J. Lin, S. Fei, Z. Gao, and J. Ding, "Fault detection for discretetime switched singular time-delay systems: an average dwell 
time approach," International Journal of Adaptive Control and Signal Processing, vol. 27, no. 7, pp. 582-609, 2013.

[9] K. Zhang, B. Jiang, and P. Shi, "Fault estimation observer design for discrete-time takagi-sugeno fuzzy systems based on piecewise lyapunov functions," IEEE Transactions on Fuzzy Systems, vol. 20, no. 1, pp. 192-200, 2012.

[10] E. K. Boukas, "Singular linear systems with delay: $\mathscr{H}_{\infty}$ stabilization," Optimal Control Applications and Methods, vol. 28, no. 4, pp. 259-274, 2007.

[11] M. Wu, Y. He, J.-H. She, and G.-P. Liu, "Delay-dependent criteria for robust stability of time-varying delay systems," Automatica, vol. 40, no. 8, pp. 1435-1439, 2004.

[12] A. T. Vemuri, M. M. Polycarpou, and A. R. Ciric, "Fault diagnosis of differential-algebraic systems," IEEE Transactions on Systems, Man, and Cybernetics Part A: Systems and Humans., vol. 31, no. 2, pp. 143-152, 2001.

[13] Z. H. Wang, M. Rodrigues, D. Theilliol, and Y. Shen, "Actuator fault estimation observer design for discrete-time linear parameter-varying descriptor systems," International Journal of Adaptive Control and Signal Processing, vol. 29, no. 2, pp. 242258, 2015.

[14] B. Boulkroune, S. Halabi, and A. Zemouche, " $H_{-} / H_{\infty}$ fault detection filter for a class of nonlinear descriptor systems," International Journal of Control, vol. 86, no. 2, pp. 253-262, 2013.

[15] H. Hamdi, M. Rodrigues, C. Mechmeche, D. Theilliol, and N. B. Braiek, "Fault detection and isolation in linear parametervarying descriptor systems via proportional integral observer," International Journal of Adaptive Control and Signal Processing, vol. 26, no. 3, pp. 224-240, 2012.

[16] M. Chadli, A. Abdo, and S. X. Ding, " $H_{-} / H_{\infty}$ fault detection filter design for discrete-time Takagi-Sugeno fuzzy system," Automatica, vol. 49, no. 7, pp. 1996-2005, 2013.

[17] S.-J. Huang and G.-H. Yang, "Fault tolerant controller design for T-S fuzzy systems with time-varying delay and actuator faults: a k-step fault-estimation approach," IEEE Transactions on Fuzzy Systems, vol. 22, no. 6, pp. 1526-1540, 2014.

[18] S. Xu and J. Lam, Robust Control and Filtering of Singular Systems, Springer, Berlin, Germany, 2006.

[19] S. Xu, P. Van Dooren, R. Stefan, and J. Lam, "Robust stability and stabilization for singular systems with state delay and parameter uncertainty," IEEE Transactions on Automatic Control, vol. 47, no. 7, pp. 1122-1128, 2002.

[20] E. Fridman, "Stability of linear descriptor systems with delay: a Lyapunov-based approach," Journal of Mathematical Analysis and Applications, vol. 273, no. 1, pp. 24-44, 2002.

[21] D. Koenig and S. Mammar, "Design of proportional-integral observer for unknown input descriptor systems," IEEE Transactions on Automatic Control, vol. 47, no. 12, pp. 2057-2062, 2002.

[22] H. Zhang, Y. Shen, and G. Feng, "Delay-dependent stability and $H_{\infty}$ control for a class of fuzzy descriptor systems with timedelay," Fuzzy Sets and Systems, vol. 160, no. 12, pp. 1689-1707, 2009.

[23] K. Mourad, S. Mansour, and T. Ahmed, "Delay-dependent stability and robust $L_{2}-L_{\infty}$ control for a class of fuzzy descriptor systems with time-varying delay," International Journal of Robust and Nonlinear Control, vol. 23, no. 3, pp. 284-304, 2013.

[24] F. Yang and Q. L. Zhang, "Delay-dependent $H_{\infty}$ control for linear descriptor systems with delay in state," Journal of Control Theory and Applications, vol. 3, no. 1, pp. 76-84, 2005.

[25] S. J. Huang, X. Q. He, and N. N. Zhang, "New results on $H_{\infty}$ filter design for nonlinear systems with time delay via T-S fuzzy models," IEEE Transactions on Fuzzy Systems, vol. 19, no. 1, pp. 193-199, 2011.

[26] C. Lin, Q.-G. Wang, T. H. Lee, and B. Chen, " $H_{\infty}$ filter design for nonlinear systems with time-delay through T-S fuzzy model approach," IEEE Transactions on Fuzzy Systems, vol. 16, no. 3, pp. 739-746, 2008.

[27] C.-H. Lien and K.-W. Yu, "Robust control for Takagi-Sugeno fuzzy systems with time-varying state and input delays," Chaos, Solitons \& Fractals, vol. 35, no. 5, pp. 1003-1008, 2008.

[28] Y. K. Su, B. Chen, C. Lin, and H. G. Zhang, "A new fuzzy $H_{\infty}$ filter design for nonlinear continuous-time dynamic systems with time-varying delays," Fuzzy Sets and Systems, vol. 160, no. 24, pp. 3539-3549, 2009.

[29] J. H. Zhang, Y. Q. Xia, and R. Tao, "New results on $H_{\infty}$ filtering for fuzzy time-delay systems," IEEE Transactions on Fuzzy Systems, vol. 17, no. 1, pp. 128-137, 2009. 


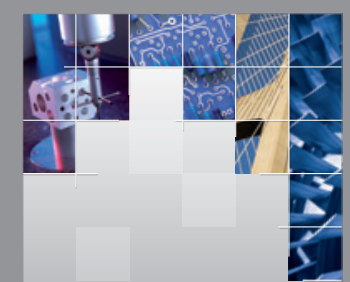

\section{Enfincering}
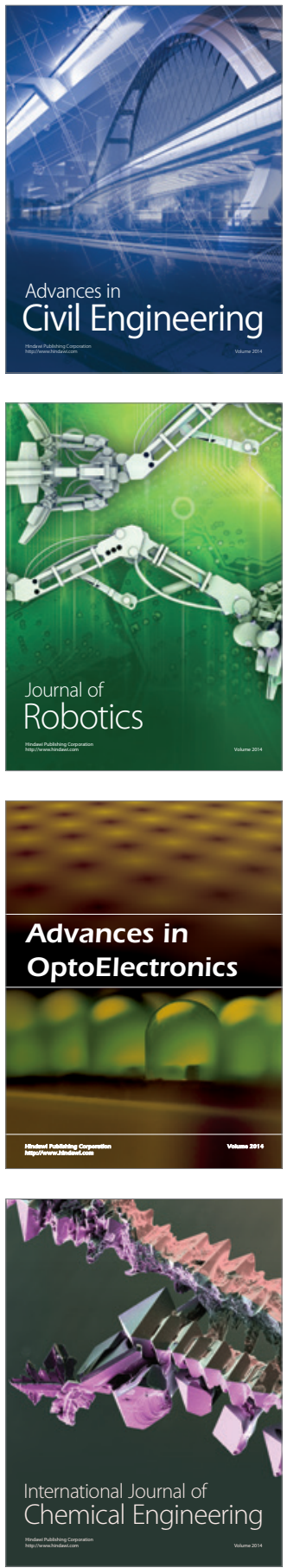

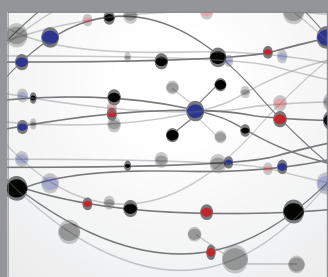

The Scientific World Journal

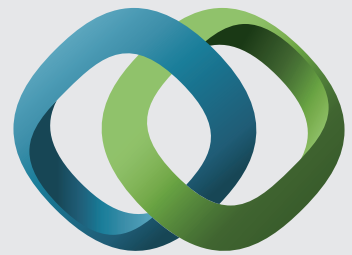

\section{Hindawi}

Submit your manuscripts at

http://www.hindawi.com
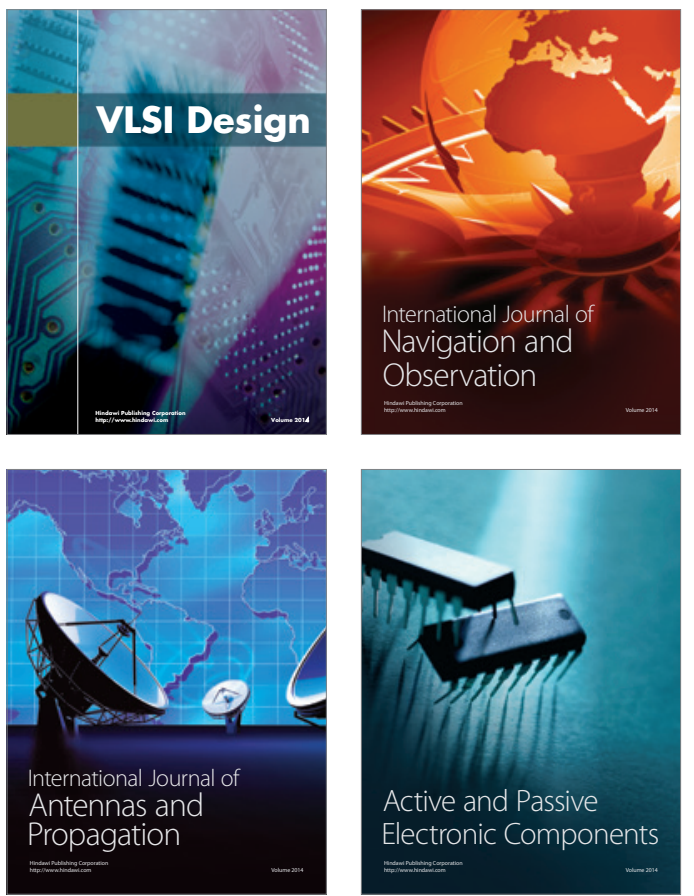
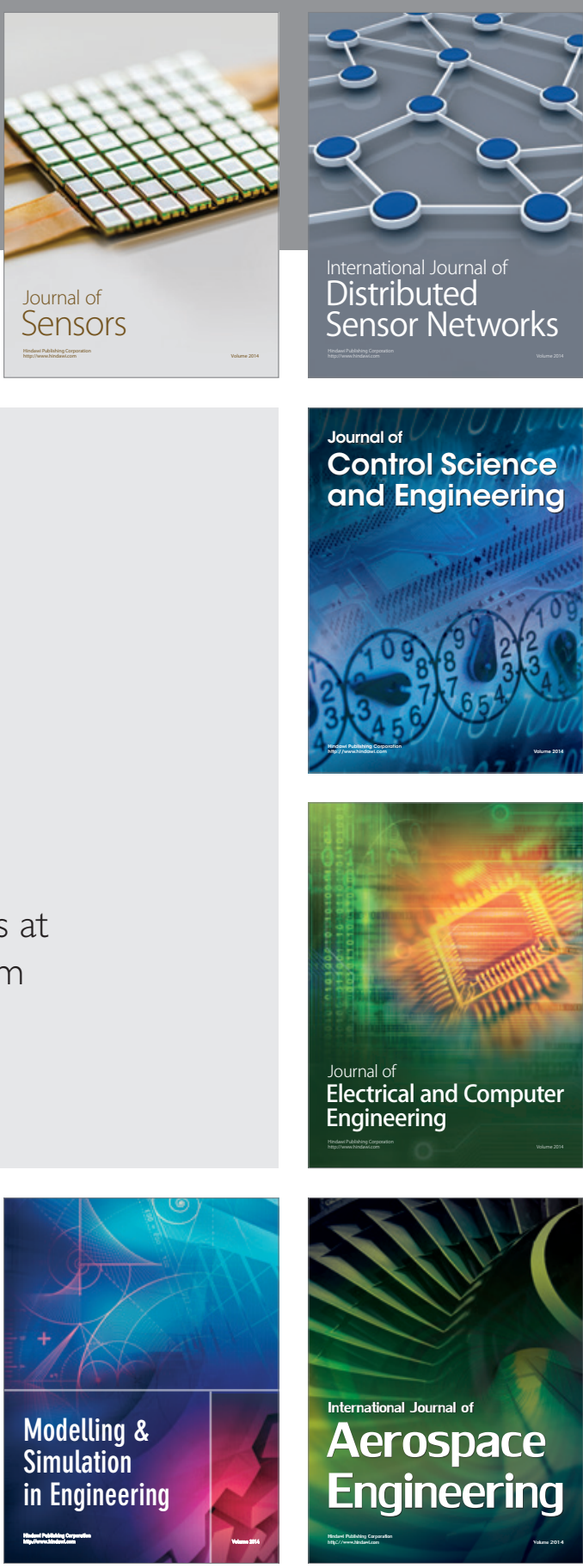

International Journal of

Distributed

Sensor Networks

Journal of

Control Science

and Engineering
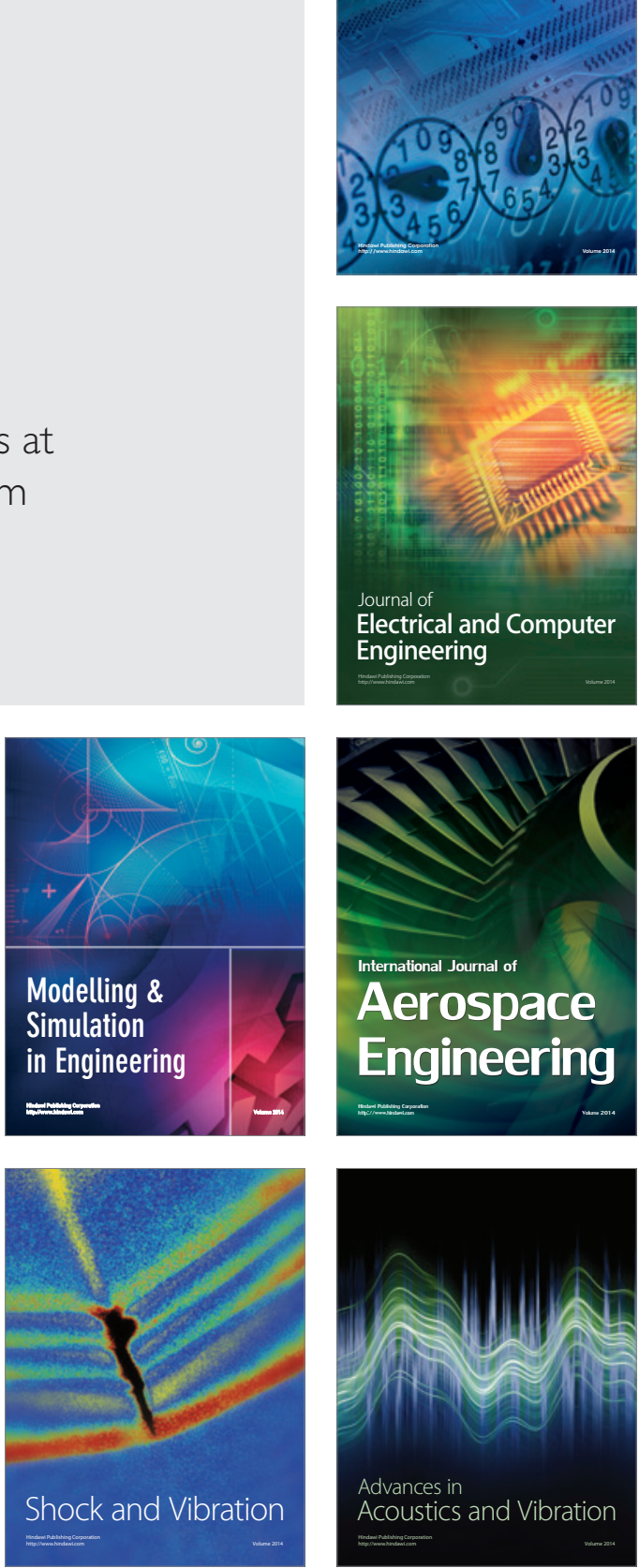